\title{
Magnetization-induced-second-harmonic generation from surfaces and interfaces
}

\author{
Andrei Kirilyuk and Theo Rasing \\ Institute for Molecules and Materials, Radboud University Nijmegen, Toernooiveld 1, 6525 ED Nijmegen, \\ The Netherlands
}

Received May 14, 2004; revised manuscript received September 21, 2004; accepted September 27, 2004

\begin{abstract}
Magnetization-induced effects in the nonlinear optical response of magnetic media, such as magnetizationinduced-second-harmonic generation (MSHG), led to very strong and novel nonlinear magneto-optical effects that appear to be very sensitive to magnetic interface properties. This surface-interface sensitivity of MSHG, in combination with the very large magneto-optical effects, has led to a fast development of this technique over the past decade. On the one hand, an extreme sensitivity of MSHG to the electronic and magnetic structure of clean surfaces has been successfully demonstrated. On the other hand, the penetration depth of light allows one to use this sensitivity to study buried interfaces in multilayer systems. Further experimental developments of the MSHG technique, such as space and time resolution as well as magnetization-sensitive-sumfrequency generation, appear to be promising as well. (c) 2005 Optical Society of America
\end{abstract}

OCIS codes: $190.4720,240.4350,260.3910$.

\section{INTRODUCTION}

Within the vast area of nonlinear optics, second-har'monic generation. (SHG) plays a very essential role. As a higher-order process, it brings new and complementary information in comparison with linear optics, partly as a result of the different selection rules for multiphoton processes and the higher-order susceptibilities involved. Another strong point of the technique is its intrinsic surface and interface sensitivity, which is derived from extremely simple yet powerful symmetry constraints. The SHG technique has therefore been widely used for studies of surfaces and interfaces. ${ }^{1-4}$

The breaking of time-reversal symmetry leads to a number of well-known magneto-optical (MO) effects such as Faraday rotation in transmission and Kerr rotation in reflection, 5 For nonlinear optics, in the electric-dipole approximation even-order effects such as SHG are allowed only in media with a broken space-inversion symmetry. As a consequence even-order nonlinear MO effects can be observed only in materials in which both space-inversion and time-reversal symmetry are broken. Although the first predictions of magnetic effects in SHG were made 40 years ago ${ }^{6-9}$ and discussed in several theoretical publications, ${ }^{10-13}$ the field of nonlinear MO really got underway in the past decade after observation of huge MO effects from magnetic surfaces and interfaces. ${ }^{14-16}$ This "revival" and recent strong development of nonlinear MO are clearly related to the enormous interest in the study and applications of magnetic multilayers and nanostructures as well as to the development of solid-state, mode-locked, femtosecond lasers that are particularly suitable for these kinds of studies. ${ }^{17}$

One of the very important fundamental achievements was the demonstration of the extreme sensitivity of magnetization-induced-second-harmonic generation (MSHG) to the slightest modifications of the spinpolarized electronic structure of transition metal surfaces. ${ }^{18-23}$ Even tiny increases in the magnetization of the surface layer caused by the presence of atoms with low coordination numbers on surfaces with atomic steps could be detected. ${ }^{21}$ On the other hand, the possibility of distinguishing the contributions from different interfaces ${ }^{24}$ showed a way to measure the magnetization of a buried interface and therefore triggered a great deal of applied interest. In addition it has been demonstrated that - confirming the original predictions-SHG can be used to study the antiferromagnetic ordering and even to image antiferromagnetic domains, which is very hard or even impossible to do with other techniques. ${ }^{25,26}$

This review is organized as follows: First a general description of MSHG is given followed by a discussion of the theory of MSHG in Section 2. Then the details of various experimental techniques are given in Section 3 . Section 4 discusses the applications of MSHG to magnetic surfaces. Section 5 is concerned with buried magnetic interfaces and is followed by a discussion of the unavoidable bulk contributions to the interface MSHG. Further developments of the technique are briefly outlined in Section 6.

\section{THEORY OF MAGNETIZATION- INDUCED-SECOND-HARMONIC GENERATION}

A. GeneraI Concept

An incident light wave induces a polarization in a medium that serves as a source for the transmitted and reflected light. This polarization $\mathbf{P}$ can be written in the electric-dipole approximation as an expansion in powers of the optical electric field $\mathbf{E}(\omega)$ :

$$
\mathbf{P}(\omega, 2 \omega, \ldots)=\chi^{(1)} \mathbf{E}(\omega)+\chi^{(2)} \mathbf{E}(\omega) \mathbf{E}(\omega)+\ldots
$$

The tensor $\chi^{(1)}$ is the linear optical susceptibility allowed in all media. SHG is described by the second term on the 
right-hand side with the corresponding nonlinear tensor' $\chi^{(2)}$ allowed only in noncentrosymmetric media. The latter is easy to verify by carrying out the inversion operation that changes the sign of every polar vector, such as $\mathbf{P}$ or E. Alternatively $\chi^{(2)}$ is allowed at surfaces or interfaces of a centrosymmetric medium, giving rise to the surface-interface sensitivity of the technique. For crystals with a spontaneous or magnetic field-induced magnetization $\mathbf{M}$, the nonlinear second-order optical polarization of a medium $P^{\text {nl }}(2 \omega)$ can be written as

$$
\mathbf{P}^{\mathrm{nl}}(2 \omega)=\chi^{\mathrm{cr}} \mathbf{E}(\omega) \mathbf{E}(\omega)+\chi^{\operatorname{magn}} \mathbf{E}(\omega) \mathbf{E}(\omega) \mathbf{M},
$$

where the first term on the right-hand side describes the purely crystallographic contribution while the second exists only in the presence of a magnetization $\mathbf{M}$ and describes MSHG. Note that $\mathbf{M}$ is an axial vector, so that the inversion operation does not change its sign, and the surface -interface sensitivity also holds for magnetic materials. Thus these two contributions to the nonlinear polarization $\mathbf{P}^{\mathrm{nI}}(2 \omega)$ are of electric-dipole character and are allowed simultaneously in noncentrosymmetric media, but their properties are different. The crystallographic contribution is described by a polar tensor $\chi^{\mathrm{cr}}$ of rank 3 , whereas the magnetization-induced contribution is described by an axial tensor $\chi^{\text {magn }}$ of rank 4 . In lossless media $\chi^{\mathrm{cr}}$ is a real tensor and $\chi^{\text {magn }}$ is an imaginary tensor; therefore there is no interference between the SHG waves coming from these two sources for linearly polarized fundamental light. ${ }^{6,12}$ The interference becomes allowed for linearly polarized fundamental light in the absorption region, because both tensors will be complex, or by using circular optical excitation. It is this interference that gives rise to new, nonlinear, $\mathrm{MO}$ effects that have no counterparts in linear optics. ${ }^{27-29}$

Equation (2) is general and fully describes MSHG in the electric-dipole approximation. The number of nonzero components of $\chi^{(\mathrm{er})}$ and $\chi^{(\mathrm{magn})}$ tensors depends on the crystallographic and magnetic symmetry of the sample. For high-symmetry systems (the vast majority of thin-film structures studied so far) each of these tensors consists of a few components only. Moreover these components are not intermixing with each other; i.e., the tensor

$$
\chi^{(2)}=\chi^{\mathrm{cr}}+\chi^{\mathrm{magn}} \cdot \mathbf{M}
$$

can be written as a single third-rank tensor whose components are either even or odd in M. An important case is that of an isotropic interface (that of a quite standard polycrystalline film, for example). In this case, and with $x z$ being the plane of incidence, the nonlinear MO tensor $\chi^{(2)}$ can be written as ${ }^{12}$ tional to it-see Ref. 30). Thus the nonlinear MO properties of an isotropic interface with a selected magnetization direction are characterized by up to 10 (complex) numbers. (The sum of 10 is the result of additional symmetry relations such as $\chi_{z x x}=\chi_{z y y}$, etc.)

Here $\mathbf{E}(\omega)$ and $\mathbf{P}(2 \omega)$ are local fields and polarizations; to relate them to the amplitudes of incoming and outgoing electromagnetic waves, appropriate Fresnel factors should be used. In fact the $\chi^{(2)}$ tensor alone determines the geometrical symmetry properties of MSHG; for an extended treatment of these properties, both theoretical and experimental, we refer the reader to Ref. 29 .

The $\chi^{(2)}$ tensor of Eq. (4) allows one to understand qualitatively the behavior of SHG as a function of the magnetization reversal. In the transverse $\mathrm{MO}$ geometry M\|y (see Fig. 1) Eq. (4) shows that in the case of $s$-polarized incident light (i.e., $\mathbf{E}_{\mathrm{in}}=\mathbf{E}_{\mathrm{y}}$ ) one even $\left(\chi_{z y y}\right)$ and one odd $\left[\chi_{x y y}\left(M_{y}\right)\right]$ tensor element will produce the outgoing second-harmonic (SH) waves. Both of them, in fact, lead to the same $p$ polarization of the output. Therefore the total MSHG output can be written as

$$
I^{2 \omega}( \pm \mathbf{M}) \propto\left|E_{\text {in }}\right|^{4}\left[\alpha \chi_{z y y} \pm \beta \chi_{x y y}(\mathbf{M})\right]^{2},
$$

where $\alpha$ and $\beta$ denote the corresponding Fresnel factors (they result for example in the angle-of-incidence dependence of the MSHG signal). Thus the reversal of $\mathbf{M}$ leads to a change in the SHG intensity.

In contrast, in the longitudinal geometry with $M \| x$ the corresponding odd element $\left[\chi_{y y y}\left(M_{x}\right)\right]$ gives rise to an $s$-polarized output. The total output polarization will thus be a vector' sum of the two orthogonal vectors, one of them being reversed upon reversal of $\mathbf{M}$. The resulting MO effect is therefore a change of SHG polarization. ${ }^{15,31}$

The problem of calculating the nonlinear (magneto-) optical response of a given medium contains two clearly distinct parts: (i) calculation of the nonlinear susceptibility and (ii) with known susceptibility, calculation of the outgoing MSHG intensity. Both these parts are nontrivial and require much attention: Part (i) involves heavy electronic structure calculations in a nonperiodic electronic system, while (ii) contains nontrivial electromagnetic boundary conditions and complicated multiplescattering processes.

\section{B. Nonlinear Magneto-Optical Susceptibility}

As the first step in the calculation of the nonlinear $\mathrm{MO}$ susceptibility tensor $\chi^{(2)}$, a complete electronic spindependent band structure of the sample should be computed, including the corresponding wave functions, Since by "sample" we mean surface or interface, i.e., nonperiodic structure, both the band structure and wave

$$
\chi^{(2)}=\left(\begin{array}{cccccc}
\chi_{x x x}\left(M_{y}\right) & \chi_{x y y}\left(M_{y}\right) & \chi_{x z z}\left(M_{y}\right) & \chi_{x z y}\left(M_{z}\right) & \chi_{x z x}^{\mathrm{cr}} & \chi_{x x y}\left(M_{x}\right) \\
\chi_{y x x}\left(M_{x}\right) & \chi_{y y y}\left(M_{x}\right) & \chi_{x z z}\left(M_{x}\right) & \chi_{y z y}^{\mathrm{cr}} & \chi_{y z x}\left(M_{z}\right) & \chi_{y x y}\left(M_{y}\right) \\
\chi_{z x x}^{\mathrm{cr}} & \chi_{z y y}^{\mathrm{cr}} & \chi_{z z z}^{\mathrm{cr}} & \chi_{z z y}\left(M_{x}\right) & \chi_{z z x}\left(M_{y}\right) & \chi_{z x y}\left(M_{z}\right)
\end{array}\right) .
$$

The elements shown to depend on $M_{i}$ are odd in the corresponding magnetization component (roughly propor- functions will be position-dependent in the direction perpendicular to the surface. 


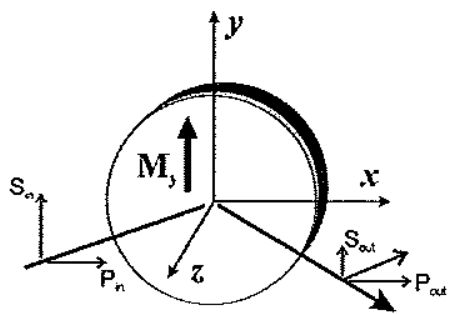

Fig. 1. Schematic geometry of the experiment: $x z$ is the plane of incidence, $\mathbf{M}$ is directed along $y$ (transversal geometry) or along $x$ (longitudinal geometry). Input light is either $p$-polarized $\left(\mathrm{E}_{x}, \mathrm{E}_{z}\right)$ or $s$-polarized $\left(\mathrm{E}_{y}\right)$.

Next, the electronic structure serves as a background for the calculation of the MO response. This has been done by Hübner and Bennemann ${ }^{13}$ and Hübner ${ }^{32}$ by use of Heisenberg's equation-of-motion formalism in the second-ordex perturbation theory. The screening of the driving electromagnetic field by the system's electrons was taken into account self-consistently.

The surface or interface susceptibility is given by the formula ${ }^{30}$ monolayer on top of a 1-, 2-, 3-, or 4-monolayer "substrate." The results indeed showed the expected large, nonlinear, MO effects. Such a sample is close to the limit of modern computation possibilities. Moreover, even here the calculation procedure was not entirely stable, as their own tests showed. The problem is that small errors in calculating the wave functions because of a restricted basis set (to save computation time) are amplified when three transition-matrix elements are multiplied to obtain the final result.

Another approach, based on the first-principles LayerKorringa-Kohn-Rostocker method, was employed to calculate the MSHG response from a Ni(110) surface. The preliminary results showed the contribution of the surface states to the nonlinear MO susceptibility ${ }^{34}$ in good agreement with the experimental results of Veenstra et $a$. $^{23}$

\section{Calculation of MSHG from Multilayers}

To calculate the MSHG response from a multilayer system one must realize that in fact the nonlinear susceptibility $\chi^{(2)}$ is expected to decrease quickly on both sides of

$$
\begin{aligned}
& \chi_{i j m}(2 \omega)=\frac{e^{3} \mathbf{q}_{1} a}{\Omega} \underset{\lambda_{\text {s.o. }}}{\hbar \omega} \sum_{\mathrm{k}, l, l^{\prime}, l^{\prime \prime}, \sigma} M_{i} M_{j} M_{m}
\end{aligned}
$$

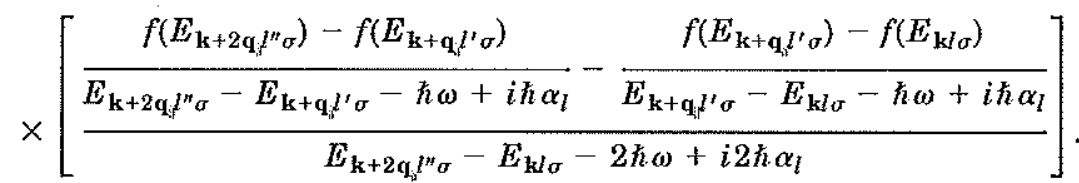

Here the symmetry of the wave functions enters through the dipole-matrix elements $M_{i}=\left\langle\Psi_{k, l, \sigma}\left|p_{i}\right| \Psi_{k, l^{\prime}, \sigma}\right\rangle$, where $p_{i}$ is the momentum operator. It is this symmetry that is responsible for the selection rules giving rise to the surface sensitivity of the response. The dependence on the electronic structure results from the eigenvalues $E_{\mathbf{k}, l, \sigma}$, which depend on the wave vector $\mathbf{k}$, the band in$\operatorname{dex} l$, and the spin $\sigma$. Note that the matrix elements may involve $s-, d$-states, as well as quantum-well states that appear in ultrathin layers, and will depend on the corresponding wave functions. $f\left(E_{\mathrm{k}, l, \sigma}\right)$ is the Fermi function and $\alpha_{l}$ is the Lorentzian broadening of the states. Taking into account only vertical transitions, Eq. (6) already shows how changes of the susceptibility result from modifications in the joint density of states, which is probed by nonlinear optics. Since SHG occurs at the surface and the interface of the film, the summation over the energy eigenvalues has to be performed according to the surface and the interface electronic structure. Thus for a paramagnetic material, the band structure for both spin directions is the same and no spin dependence results. In the case of a ferromagnetic material, the nonlinear tensor $\chi_{i j m}(\mathbf{M})$ will be separated into odd and even components as discussed above.

The only attempt to carry out the entire computational procedure was made by Andersen and Hübner, ${ }^{33}$ who calculated the nonlinear MO response of a single $\mathrm{Fe}(001)$ the interface. As a result the spatial distribution of $\chi^{(2)}$ can be well approximated by an infinitesimally thin nonlinear sheet between the two "non-nonlinear" media (see Fig. 2) ${ }^{35-38}$ This is even more the case because the characteristic length to compare is the wavelength of light, typically several hundred nanometers. Such an approximation reduces the number of parameters in the problem. A well-known concept in optics is to treat the boundary conditions at an interface and the propagation of light through a homogeneous slab in terms of matrices that relate the field components on both sides of the interface and the layer, respectively. Describing the full multilayer is thus reduced to a simple matrix multiplication. ${ }^{39}$

Because of the very small nonlinear susceptibility values, the total problem can be bisected. In the first part the influence of the nonlinearity on the light behavior is totally neglected. The local electric fields and polarizations induced by the incident light inside the structure are derived from the primary electric field through the linear susceptibility tensor. The tensor; in tum, may depend on the layer magnetization, thus giving rise to linear MO effects. The induced polarizations are actually quite small compared with the fundamental electric field and can be considered perturbations.

The second part of the problem concerns the electromagnetic waves at the SH frequency. Here the funda- 
mental electromagnetic field is absent and the $2 \omega$ polarizations play the role of the only sources. If all the layers of the structure possess a center of inversion, then within the electric-dipole approximation, there will be no volume polarization. The total SH yield can thus be related to the interfaces only, each interface being described by its own nonlinear optical tensor $\chi^{(2)}$.

It should be noted that even in linear optics, the normal component of the electromagnetic field is discontinuous across ideal interfaces. The surface polarizations become radiating sources. The corresponding electric fields can be obtained from Maxwell's equations, which are now accompanied by the unconventional boundary conditions' taking into account singular polarizations of ideal interfaces. $^{1}$ A comprehensive treatment of problems related to the model of both linear and nonlinear MO effects in multilayer's has been given by Atkinson and Kubrakov. ${ }^{40}$ Most often, however, bulk anisotropy of layers can be neglected, together with the linear MO effects that are argued to be small enough.

The parameters accessible experimentally are the SHG intensity and polarization. In addition the phase of the total SHG output can be determined quite straightforwardly. ${ }^{41,42}$ To determine the nonlinear susceptibilities at interfaces, the MSHG output is first measured as a function of some parameters, e.g., angle of incidence, magnetization, sample thickness, azimuthal angle, etc. Then multiple-scattering calculations are used to fit the obtained data by use of the nonlinear optical tensor components as fitting parameters, provided that the obtained experimental data are sufficient for an unambiguous fit. Various polarization combinations help to distinguish different tensor components by selectively exciting one or another combination of them. An example of such an analysis will be included in Section 5 .

The main assumption of the model discussed is that it considers the source of the SH field in the form of an infinitely thin, coherently and homogeneously polarized sheet, as shown in Fig. 2. Although this assumption is justifiable in many cases investigated experimentally, it is not sufficiently general. Moreover, it is based on a macroscopiclike description of the source of the SH field that does not provide a direct physical insight into the processes involved in the SHG phenomena.

A different model was discussed recently ${ }^{43}$ in which the problem of the SHG was considered from a different point

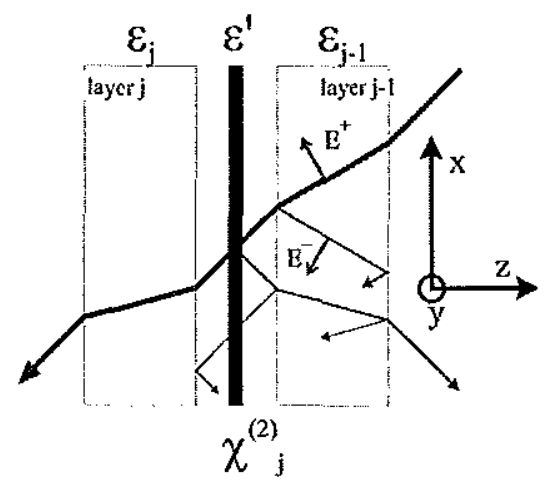

Fig. 2. Infinitesimal nonlinear sheet inside an artificial vacuum sheet at an interface. of view that can be better related to the microscopic description of the origin of the SH field. The SH field is considered to be generated by a point electric dipole oscillating at an angular frequency $2 \omega$ and positioned at the layer interface. This is closely related to the symmetry considerations used in the macroscopic models such as that discussed above.

This approach to the SHG has some advantages. In particular once the electromagnetic field generated by the point dipole is known, it can be used to evaluate the $\mathrm{SH}$ field generated from a system with arbitrarily spatially distributed dipoles. Furthermore, as it is based on a well-understood microscopic quantity (an elementary dipole), it can be more directly related to a quantummechanical description of SHG.

The elegance of the model developed becomes apparent when systems with an inhomogeneous distribution of the susceptibility tensors along the interfaces are considered. This is the case when the magnetization of the layers, and thus the interfaces, exhibits variation in the lateral dimension due for example to the presence of magnetic domains, propagating spin waves, periodic structures, magnetic nanostructures, etc., and where the optical properties (layer thicknesses and permittivity tensors) can be assumed to be laterally homogeneous. ${ }^{43}$

\section{Interface and Bulk Contributions to MSHG from a Superlattice}

It would be misleading to state that all SHG response is generated at interfaces only. Strictly speaking, the surface nonlinear optical tensor $\chi$ does not even fulfill the energy conservation law, one of the basic principles of physics. Re-examined closely, ${ }^{44}$ this paradoxical result can be understood with the aid of an additional bulk contribution to the energy flow, as part of the surface response appears to be determined by the bulk parameters alone.

Having realized that, it is interesting to look more closely at the description of surface and bulk contributions to MSHG from a multilayer. The polarization $\mathbf{P}$ can be written as an expansion in powers of the optical electric field $\mathbf{E}(\omega)$ :

$$
\begin{aligned}
\mathbf{P}(\omega, 2 \omega, \ldots)= & \chi^{1, d} \mathbf{E}(\omega)+\chi^{1, q} \nabla \mathbf{E}(\omega) \\
& +\chi^{2, d} \mathbf{E}(\omega) \mathbf{E}(\omega)+\chi^{2, q} \mathbf{E}(\omega) \nabla \mathbf{E}(\omega) \ldots
\end{aligned}
$$

The tensor $\chi^{1, d}$ is the linear optical susceptibility allowed in all media. SHG is described by the third and the fourth terms, where the electric-dipole tensor $\chi^{2, d}$ is allowed only in noncentrosymmetric media and at surfaces and interfaces, while the quadrupole tensor $\chi^{2, q}$ is allowed everywhere. For crystals with a spontaneous or magnetic-field induced magnetization $\mathbf{M}$, expansion of the nonlinear optical polarization of a medium $\mathbf{P}^{\text {nl }}(2 \omega)$ can be further written (keeping only linear-in-magnetization terms) as

$$
\begin{aligned}
\mathbf{P}^{\mathrm{nl}}(2 \omega)= & \chi^{\mathrm{cr}} \mathbf{E}(\omega) \mathbf{E}(\omega)+\chi^{\mathrm{m}} \mathbf{E}(\omega) \mathbf{E}(\omega) \mathbf{M} \\
& +\chi^{q, \mathrm{cr}} \mathbf{E}(\omega) \nabla \mathbf{E}(\omega)+\chi^{q, \mathrm{~m}} \mathbf{E}(\omega) \nabla \mathbf{E}(\omega) \mathbf{M}
\end{aligned}
$$


where the first and third terms on the right-hand side describe the purely crystallographic contributions, while the second and fourth exist only in the presence of magnetization $\mathbf{M}$.

Although smaller, the last two terms in Eq. (8) originate from the bulk and therefore may be comparable in magnitude to the strong dipole contribution coming from the very thin interface layer. Experimental results (see below) confirm this assumption and substantiate the necessity to take this contribution into account in highquality, single-crystalline multilayers.

The structure of choice is a (001)-oriented multilayex, often met in practice. ${ }^{45}$ The crystallographic electricdipole contribution from such structure with fourfold symmetry (that corresponds to the $4 / \mathrm{mmm}$ point-group symmetry) is described by a third-rank tensor and is therefore isotropic. On the other hand, both magnetic electric-dipole and crystallographic electric-quadrupole terms give an anisotropic response, indicating the presence of higher-order contributions.

\section{Local Contributions}

In a superlattice one has the top surface and many interfaces that can contribute to the second-order response. Note however that there is a strong cancellation expected between neighboring interfaces $I_{1}$ and $I_{2}$ due to their opposite orientation, leading to

$$
\chi^{(2), I_{1}} \approx-\chi^{(2), I_{2}},
$$

where $\chi^{(2), I_{1}}$ and $\chi^{(2), I_{2}}$ are the nonlinear susceptibilities of the two interfaces. A nonvanishing contribution of the interfaces to the MSHG response can then arise as a result of two factors:

1. The cancellation may be incomplete, $\chi^{(2), I_{1}}$ $+\chi^{(2), I_{2}} \neq 0$, because of a slight difference between the "upward" and "downward" interfaces as a result, e.g., of growth-induced variations of the crystallographic structure of the two types of interfaces. Also, because of the extended character of the electron wave functions, the top surface may induce effects on the electronic structure of buried interfaces that can be different for $I_{1}$ and $I_{2}$.

2 . The fully antisymmetric part of the interface susceptibilities $\left[\chi^{(2), I_{1}}-\chi^{(2), I_{2}}\right] / 2$ can also contribute to the total response as a result of a small difference in the local optical fields that are retarded and attenuated at the lower interface.

Since the thickness of the layers in the superlattice is very small relative to the optical wavelength, one can introduce macroscopically averaged fields and nonlinear polarizations and replace the interior of the sample by a uniform medium with effective parameters. Within this effective-medium approach the contributions to the MSHG response can then be described in terms of (i) dipolelike susceptibility $\chi^{S}$ of the top surface, (ii) dipolelike susceptibility $\chi^{l, d}$ due to incomplete cancellation of the interface susceptibilities, and (iii) a nonlocal (quadrupolelike) contribution that arises from the fully antisymmetric part of the interface susceptibilities as a result of spatial variation of the macroscopic effective field $\mathbf{E}^{\mathrm{eff}}(\omega)$ along $z$ :

$$
P_{i}^{I, Q}(2 \omega)=\chi_{i j z l}^{l, Q} E_{j}^{\mathrm{eff}}(\omega) \nabla_{z} E_{l}^{\mathrm{eff}}(\omega) .
$$

Note that the derivatives of $\mathbf{E}^{\mathrm{eff}}(\omega)$ along the layers do not enter into Eq. (10) since within the plane-wave approximation the spatial variation of $\mathbf{E}^{\mathrm{eff}}(\omega)$ in the tangential direction is fully determined by the tangential projection of the wave vector of the incident wave through the whole sample. In this section we focus on the dipolelike contributions of the top surface and interfaces, while the contribution due to Eq. (10) is discussed below in Subsection 2.D.2.

Within the plane-wave approximation the amplitude of the $\alpha$-polarized MSHG response induced by $\beta$-polarized fundamental light $(\alpha, \beta=s, p)$ arising through the dipolelike nonlinear susceptibilities can be written as

$$
E_{2 \omega}^{\alpha, \beta}=\sum_{i^{\prime}, j^{\prime}, k^{\prime}} \chi_{i^{\prime} j^{\prime} k^{\prime}}, \tilde{F}_{i}^{\alpha}(2 \omega) F_{j^{\prime}}^{\beta}(\omega) F_{k^{\prime}}^{\beta}(\omega) E_{0}^{\beta}(\omega)^{2}
$$

where $\tilde{F}_{i}^{\alpha}(2 \omega)$ and $F_{j^{\prime}}^{\beta}(\omega)$ are the Fresnel factors, $E_{0}(\omega)$ is the amplitude of the incident wave, and $\chi_{i^{\prime} j^{\prime} k^{\prime}}$ denotes elements of the total effective dipole susceptibility of the top surface and the interfaces in the laboratory frame.

As before the effect of the magnetization on the Fresnel factor's is neglected. The Fresnel factors can also be assumed isotropic (independent of $\phi$ ) so that $F_{y^{\prime}}^{p}=F_{x^{\prime}}^{s}$ $=F_{z}^{s}=0$ and the dependencies on the azimuthal angle $\phi$ and the direction of the magnetization $\mathbf{M}$ arise solely from the nonlinear susceptibility $\chi_{i^{\prime} j^{\prime} k^{\prime}}(\phi, M)$. We now rewrite the expansion of Eq. (3) in the following way:

$$
\chi_{i j k}(\mathbf{M})=\chi_{i j k}(0)+X_{i j k l} M_{l},
$$

where $X$ is an axial fourth-rank tensor and we neglect the higher-order terms.

For a fourfold symmetric sample the nonmagnetic part $\chi(0)$ of the susceptibility possesses three independent elements with $z z z, z\|\|$, and \|\|$z$ indices, where the $\|$ stand for the in-plane $x$ or $y$ coordinates. This tensor is purely isotropic and does not lead to rotational anisotropy. Its contribution to the $s$-polarized response vanishes for both $p$ - and $s$-polarized fundamental waves $(p, s$ and $s, s$ response, respectively). All three components of the $\chi(0)$ tensor contribute to the response for the $p, p$ polarization combination while only the $z\|\|$ element contributes to the $s, p$ response.

In the longitudinal geometry the magnetization vector is within the surface plane. Thus in the crystallographic frame we need the components of the tensor $X_{i j k l}$ with $l$ $=x$ or $y$. For a fourfold symmetric sample they are ${ }^{46}$

$$
\begin{array}{ll}
X_{x x y x}=-X_{y y x y}, & X_{y x x x}=-X_{x y y y}, \\
X_{y y y x}=-X_{x x x y}, & X_{y z z x}=-X_{x z z y}, \\
X_{z z y x}=-X_{z z x y} . &
\end{array}
$$

The elements connected to the first and the last elements in Eqs. (13) by the simple permutation symmetry $X_{i j k l}$ $\equiv X_{i k j l}$ are omitted.

From the above it is straightforward to derive ${ }^{45}$ that the azimuthal dependence of the response has the form

$$
E_{2 \omega}^{p, p}\left(\phi, \pm \mathbf{M}_{l}\right)=A^{p, p} \pm C^{p, p} \sin 4 \phi,
$$




$$
\begin{aligned}
& E_{2 \omega}^{s, p}\left(\phi, \pm \mathbf{M}_{l}\right)=A^{s, p} \pm C^{s, p} \sin 4 \phi, \\
& E_{2 \omega}^{p, s}\left(\phi, \pm \mathbf{M}_{l}\right)= \pm A^{p, s} \pm B^{p, s} \cos 4 \phi, \\
& E_{2 \omega}^{s, s}\left(\phi, \pm \mathbf{M}_{l}\right)= \pm A^{s, s} \pm B^{s, s} \cos 4 \phi,
\end{aligned}
$$

where the \pm sign indicates those terms that change their sign on magnetization reversal, and $A, B$, and $C$ are independent of $\phi$ and the direction of the longitudinal magnetization $\mathbf{M}_{l}$. Note that the light intensity is related to the field through $I_{2 \omega}^{\alpha, \beta}=c /(2 \pi)\left|E_{2 \omega}^{\alpha, \beta}\right|^{2}$.

\section{Nonlocal Contributions}

To the first order in nonlocality (quadrupole) the effective nonlinear polarization is proportional to the gradient of the fundamental field:

$$
P_{i}(2 \omega)=\chi_{i j k l}^{Q}(\mathrm{M}) E_{j}(\omega) \nabla_{k} E_{l}(\omega) .
$$

This nonlinear source includes the nonlocal contributions from the interior of the layers and the fully asymmetric part of the response of the interfaces of Eq. (10) as discussed in Subsection 2.D.1. As with Eq. (12), we expand the $\chi_{i j k l}^{Q}(\mathbf{M})$ as

$$
\chi_{i j k l}^{Q}(\mathbf{M})=\chi_{i j k l}^{Q}(0)+X_{i j k l m}^{Q} M_{m}+\cdots .
$$

Below we take only the first (nonmagnetic) term of the expansion as it provides enough freedom to describe fully the experimental data. Thus in this section we completely neglect the effect of the magnetic order on the nonlocal contribution and omit "(0)" for notation simplicity.

For the $4 / \mathrm{mmm}$ symmetry class the independent elements of the $\chi^{Q}$ tensor are

$$
\begin{array}{lll}
\chi_{x x y y}^{Q}=\chi_{y y x x}^{Q}, & \chi_{x y x y}^{Q}=\chi_{y x y x}^{Q}, & \chi_{x x x x}^{Q}=\chi_{y y y y}^{Q}, \\
\chi_{x x z z}^{Q}=\chi_{y y z z}^{Q}, & \chi_{z z x x}^{Q}=\chi_{z z y y}^{Q}, & \chi_{x z x z}^{Q}=\chi_{y z y z}^{Q}, \\
\chi_{z x z x}^{Q}=\chi_{z y z y}^{Q}, & \chi_{z z z z}^{Q} .
\end{array}
$$

Note that the fourfold anisotropy may arise only from the elements in the first row with purely tangential components, while the other components do not change on azimuthal rotation.

As in Subsection 2.D.1 (see Ref. 45) one can see that the azimuthal dependence of the response field amplitude now reads as

$$
\begin{aligned}
& E_{2 \omega}^{p, p}\left(\phi, \pm \mathbf{M}_{l}\right)=A^{p, p}+B^{p, p} \cos 4 \phi \pm C^{p, p} \sin 4 \phi, \\
& E_{2 \omega}^{s, p}\left(\phi, \pm \mathbf{M}_{l}\right)=A^{s, p}+B^{s, p} \cos 4 \phi \pm C^{s, p} \sin 4 \phi, \\
& E_{2 \omega}^{p, s}\left(\phi, \pm \mathbf{M}_{l}\right)= \pm A^{p, s} \pm B^{p, s} \cos 4 \phi+C^{p, s} \sin 4 \phi, \\
& E_{2 \omega}^{s, s}\left(\phi, \pm \mathbf{M}_{l}\right)= \pm A^{s, s} \pm B^{s, s} \cos 4 \phi+C^{s, s} \sin 4 \phi .
\end{aligned}
$$

\section{EXPERIMENTAL DETAILS}

The strong development of nonlinear magneto-optics in the past decade is also related to the development of solidstate, mode-locked lasers that combine short pulse lengths with high repetition rates and allow the study of ultrathin magnetic films without destroying them. For most MSHG experiments nowadays, a Ti:sapphire laser (82 $\mathrm{MHz}$ with 100 -fs pulses) tunable from $750-1100 \mathrm{~mm}$ but extendable to $400 \mathrm{~nm}-3 \mu \mathrm{m}$ with a parametric amplifier is used. After proper filtering the generated specular harmonic light can be analyzed.

For each polarization combination, the total MSHG response from a magnetic material can be simplified by

$$
\mathbf{P}(2 \omega)=\left[\chi_{\text {eff }}^{\text {even }} \pm \chi_{\text {eff }}^{\text {odd }}( \pm \mathbf{M})\right] E_{\omega}^{2},
$$

where $\chi_{\text {eff }}^{\text {even }}$ and $\chi_{\text {eff }}^{\text {edd }}$ are effective tensor components that are even and odd in the magnetization and describe the crystallographic and magnetic contributions to the total response, respectively. As both these contributions are complex quantities, the total (MSHG) signal is thus given by

$$
I^{2 \omega}=\left|\chi_{\text {eff }}^{\text {even }}\right|^{2}+\left|\chi_{\text {eff }}^{\text {odd }}\right|^{2} \pm 2\left|\chi_{\text {eff }}^{\text {even }}\right|\left|\chi_{\text {eff }}^{\text {odd }}\right| \cos \Delta \Phi,
$$

where $\Delta \Phi$ is the phase difference between the two contributions. The importance of the latter is obvious: When $\Delta \Phi=\pi / 2$ the interference term is zero, and changing the magnetization direction will have no effect on the total MSHG signal.

Though generally phase information is lost in intensity measurements, fortunately the phase can be measured quite easily in nonlinear optics by using interference techniques. ${ }^{47}$ The latter can also be exploited in the case where there is only a purely odd response by adding a nonmagnetic reference signal, as it is the interference between even and odd terms that gives rise to the nonlinear MO effects.

While MSHG signals give large relative MO effects, because it is a nonlinear optical technique the absolute intensities are rather small $\left(10-10^{4}\right.$ photons/sec) but easily detectable with modern photon-counting or chargecoupled devices (CCD), though care should be taken to filter out the $2 \omega$ signal versus the much stronger fundamental signal at $\omega$ (see Fig. 3).

Because of the simplicity of the experimental configuration coupled with the large effects, the transverse geometry is often used for experimental studies. One can then define a magnetic contrast or asymmetry as

$$
\begin{aligned}
A & =\frac{I^{2 \omega}(+\mathbf{M})-I^{2 \omega}(-\mathbf{M})}{I^{2 \omega}(+\mathbf{M})+I^{2 \omega}(-\mathbf{M})} \\
& =\frac{2\left|\chi_{\text {eff }}^{\text {odd }}\right| /\left|\chi_{\text {eff }}^{\text {even }}\right|}{1+\left|\chi_{\text {eff }}^{\text {even }} / \chi_{\text {eff }}^{\text {even }}\right|^{2}} \cos \Delta \Phi .
\end{aligned}
$$

Because $A$ is normalized with respect to the total SHG intensity, it does not depend on the intensity or shape of the fundamental light pulses nor on the spectral properties of optical components such as filters in the optical setup. Together with the already-mentioned simplicity, this makes $A$ a useful parameter for quantitative investigations. One should however realize that the appearance of large effects that result from the large magnetic tensor components also means that, in contrast to most linear MO effects, the nonlinear effects are often not simply linearly proportional to the magnetization, as directly follows from Eqs. (20) and (21). This can, for example, strongly affect the shape of an MSHG loop ${ }^{48}$ (see also Subsection 5.C). 


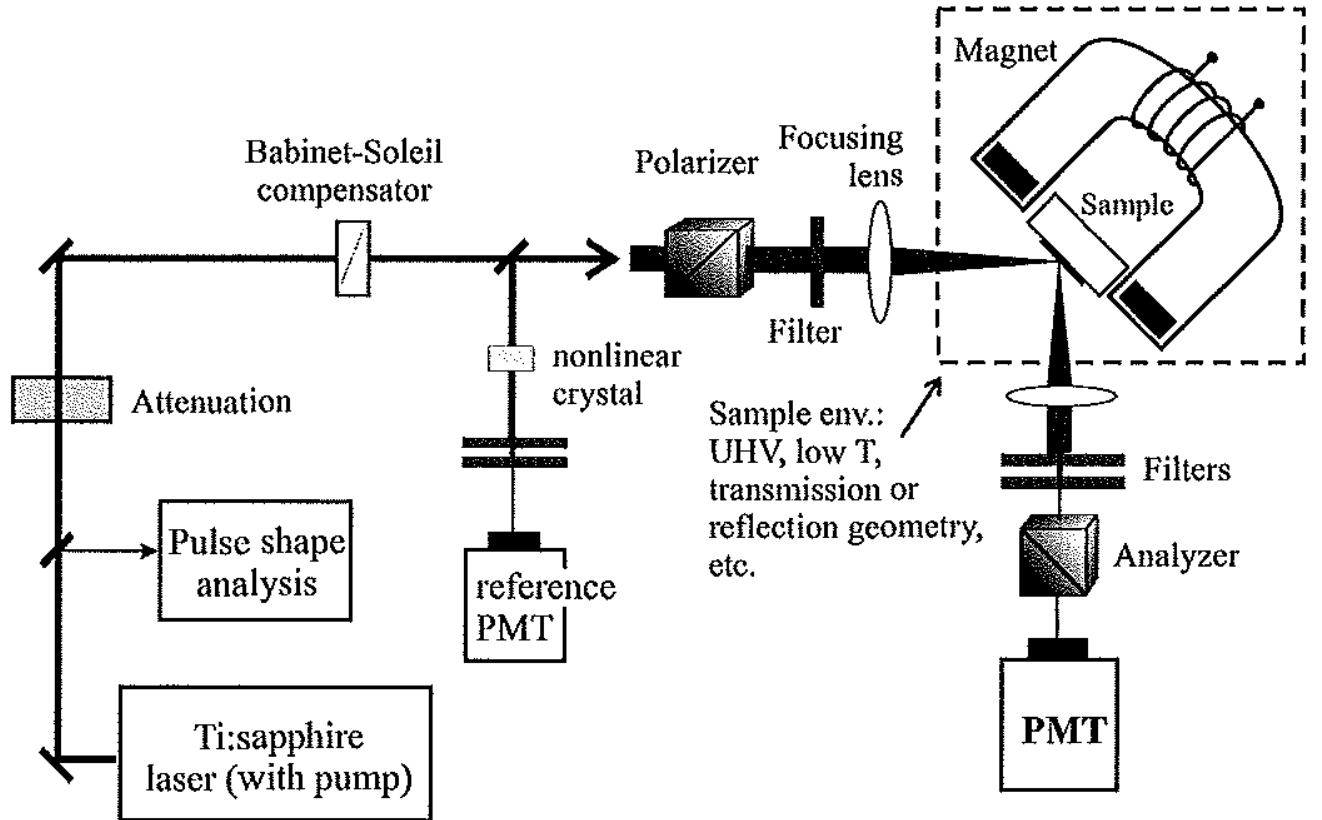

Fig. 3. Typical MSHG experimental setup with an autocorrelator for pulse shape characterization and a reference SHG channel.
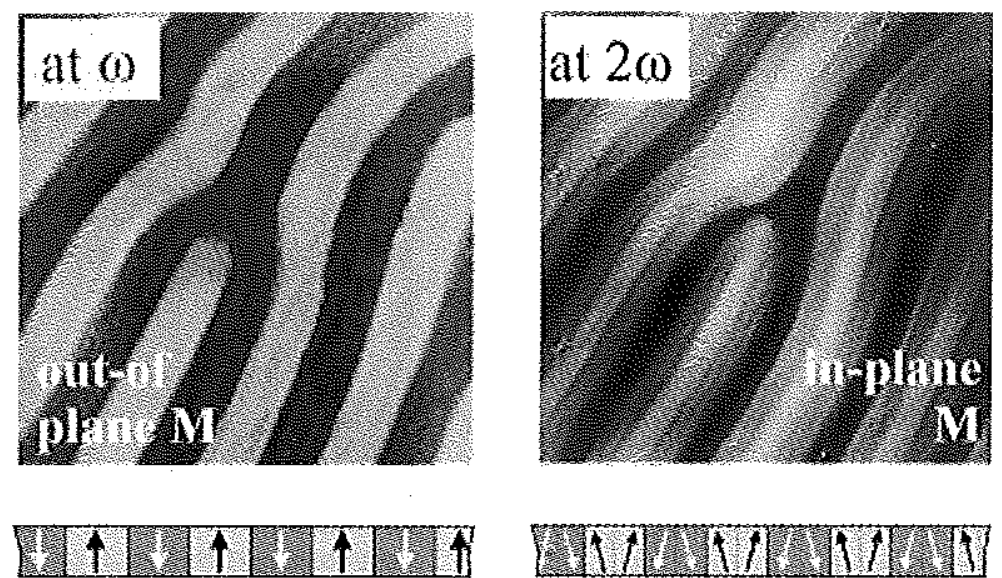

Fig. 4. Images of magnetic domain structure in a thin layer of magnetic garnet: left panel, linear Faraday effect; right panel, MSHG in transmission. Below the images, the domain structure is shown as inferred from the pictures: Faraday effect sees only the polar component of $\mathbf{M}$, while MSHG is able to detect also the in-plane component of the magnetization, showing that each up-down domain in the right panel is actually divided into two subdomains with tilted magnetization directions.

\section{A. MSHG Microscopy}

It is interesting as well as challenging to employ the nonlinear optical effects in the imaging mode. As a result of its high sensitivity to the symmetry and order, SHG has been used to image ferroelectric domains and domain walls, ${ }^{49-52}$ molecular surface ordering, ${ }^{53,54}$ and metal ${ }^{55}$ and semiconductor ${ }^{56}$ surface structures. In addition, because of its high contrast SHG microscopy could be combined with optical near-field imaging, ${ }^{57-59}$ thus expanding the resolution possibilities.

The MSHG technique can be straightforwardly adopted to provide spatial information on the magnetization distribution at interfaces. Because of its high MO contrast, as well as some rather unusual MO effects, it can also be used as a technique complementary to the standard (magneto-optical Kerr effect) MOKE microscopy.

The latter has been clearly demonstrated in thin films of magnetic garnets where the initial inversion symmetry of the bulk has been broken by the anisotropic film growth. The resulting strong MSHG response has different symmetry properties than the corresponding linear MO effects and was thus useful in distinguishing the complementary magnetization components as shown in Fig. $4 .{ }^{60}$

For imaging, laser light is focused onto a sample. The generated SHG light is used, after proper filtering, for the imaging of the sample with the help of a CCD camera. Because of the very large contrast, the images could be obtained directly and without any constrast improvement nor background subtraction, a procedure that is usually necessary to obtain the image with linear MO. The size of the MSHG image is restricted to the diameter of the focused laser beam $\approx 30 \mu \mathrm{m}$ in this case-whereas the resolution is determined by a standard criteria for the microscope used. The size limitation for the image can be overcome by using a sample-scanning procedure. Figure 
5 shows a millimeter-size image of an ultrathin stepped film in which, because of electronic quantization effects, one can observe a strong contrast from monolayer-high steps. $^{61}$ A more elaborate procedure demonstrated by Pavlov et $a l .{ }^{62}$ involves a combination of high-resolution imaging with scanning the sample and a subsequent reconstruction of the total image from small parts.

For the purposes of the present review, it is more interesting to consider MSHG imaging based on the pure interface response, such as that from $\mathrm{CoNi}-\mathrm{Pt}$ interfaces. ${ }^{63}$ Such sputtered $\mathrm{CoNi}-\mathrm{Pt}$ multilayers were found to be a promising MO material ${ }^{64}$ because of their low Curie temperature and strong $\mathrm{MO}$ effects.

Figure 6 shows the magnetization reversal process in a 9-nm-thick CoNi film starting from a fully saturated sample [Fig. 6(a)]. A magnetic field of the same value 60 Oe) was applied for the given time intervals (usually $20 \mathrm{~s}$ ) followed each time by an image accumulation in zero field (10 min per image). In the MSHG images of Fig. 6 appear some details of the domain structure with a weaker contrast than that of the opposite domains. For example, images (d) and (e) clearly show several faint stripes extending along the diagonal of the images that are possibly related to interface effects. All of them disappear later in a completely saturated sample (f). Due to the low quality of the linear MOKE images in such transverse geometry, a precise comparison of the linear and MSHG images is difficult. Therefore it was impossible (unfortunately) to give an unambiguous interpretation of these structures as interface-related.

\section{B. Measurements of the Optical Phase}

In most of the surface SHG experiments only the intensity of the SH light is routinely measured. The phase of SHG does, however, contain valuable information for a correct interpretation of the experimental data. ${ }^{23,41}$ Furthermore, phase-sensitive measurements are especially useful in surface-specific SHG where the response originates mainly from a thin surface region, so that the optical phase is directly related to the phase of the components of the surface nonlinear susceptibility $\chi^{(2)}$. As an example, the phase may give direct information about absolute molecular orientation on surfaces ${ }^{65}$ and in liquid crystals. $^{66}$ For MSHG the phase between the even and odd susceptibility components is an extremely important parameter, as it determines the actual contrast in the MSHG signal [see Eq. (21)].

The phase of the SHG response can be determined with an interference technique described in Ref. 67. This method, however, is not compatible with, e.g., ultrahigh- vacuum apparatus when femtosecond lasers are used. The reason is the dispersion of the optical windows $\left(\Delta n_{\text {glass }} \approx 10^{-2}\right)$ causes a too-large time delay $(\tau \approx 1 \mathrm{ps})$ between the fundamental and SHG pulses that destroys the interference. The following alternative approach overcomes this problem in an elegant way. ${ }^{41}$

When $2 \omega$ and $\omega$ pulses propagate through air; the relative phase $\Phi$ between them gradually changes:

$$
\Phi(d)=\Phi_{0}+\delta \Phi=\Phi_{0}+\frac{4 \pi \Delta n_{\text {air }}}{\lambda} d,
$$

where $\Delta n_{\text {air }}=n(2 \omega)-n(\omega) \approx 10^{-5}$ is the dispersion of the ambient air, $d$ is the distance the two pulses have traveled through air, and $\lambda$ is the fundamental wavelength. By using an additional SHG source (called reference) at position $d$ in the path of the beam, interference can be observed in the detected total intensity

$$
\begin{aligned}
I_{2 \omega, \mathrm{tot}}(d)= & I_{2 \omega, \mathrm{s}}+I_{2 \omega, \mathrm{r}}+2 \alpha\left(I_{2 \omega, \mathrm{s}} I_{2 \omega, \mathrm{r}}\right)^{1 / 2} \\
& \times \cos \left[\delta \Phi(d)+\Phi_{0}\right]
\end{aligned}
$$
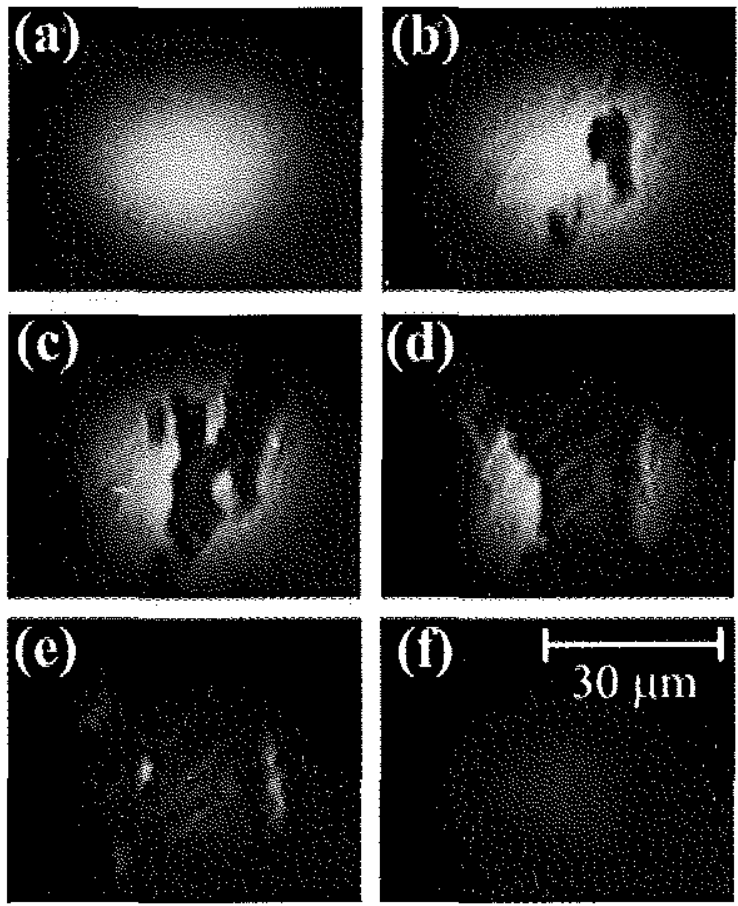

Fig. 6. MSHG images of magnetization reversal in a Pt$\mathrm{CoNi}-\mathrm{Pt}$ sandwiched layer. Magnetization is saturated "up" in image (a) and "down" in image (f). The laser spot diameter is approximately $30 \mu \mathrm{m}$. No background subtraction of any kind has been used.
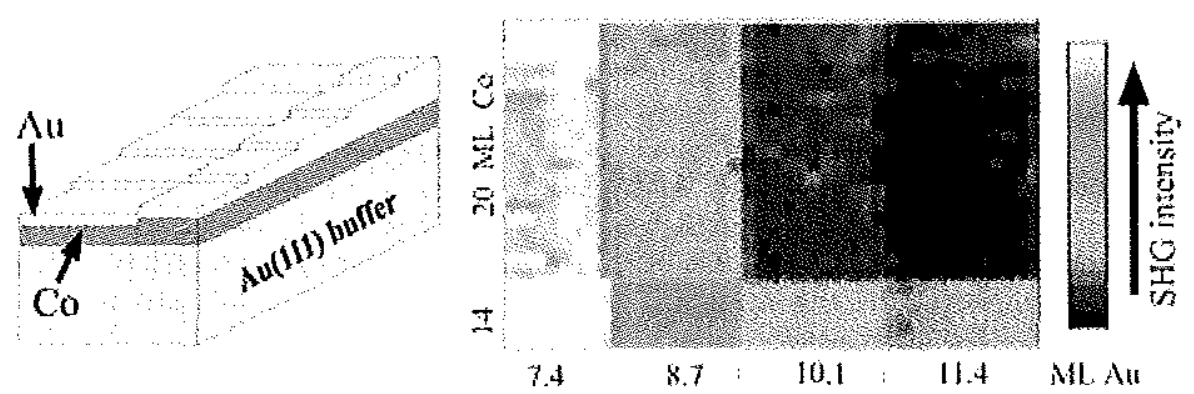

Fig. 5. Large-scale image of a stepped $\mathrm{Au}-\mathrm{Co}-\mathrm{Au}(111)$ layer showing monolayer sensitivity of SHG imaging. 
(a)

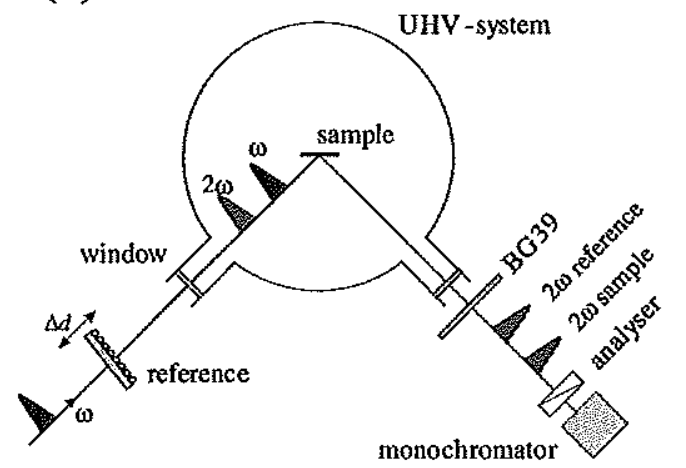

(b)

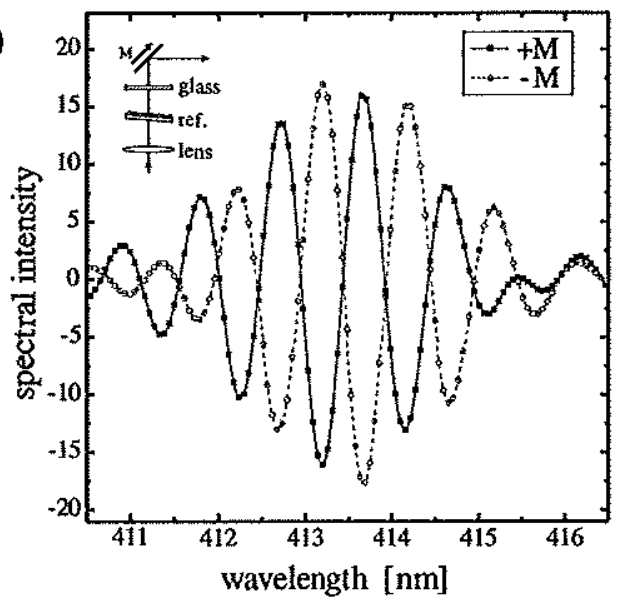

Fig. 7. (a) Sketch of the experimental setup for the spectral phase measurements. The SH pulse from the reference is delayed with respect to the fundamental laser pulse as a result of the dispersion of the window. (b) Spectral interference from a Rh-Co-Ca multilayer for two opposite magnetization directions. Reproduced from Ref. 41.

where $I_{2 \omega, \mathrm{s}}$ and $I_{2 \omega, \mathrm{r}}$ are the SHG signals generated by the sample and the reference, respectively. The spatial coherence is described by the coherence parameter $\alpha$. This interference disappears if the pulses do not overlap in time. As is shown in Fig. 3(a), this may happen if the femtosecond fundamental and SH pulses have to travel through a strongly dispersive element such as an optical window.

Looking at the problem more closely in the time domain, the optical field at the detector created by two pulses with a delay $\tau$ can be described by the function

$$
\begin{aligned}
E_{2 \omega}(t)= & E_{1} g(t) \exp \left(-i 2 \omega_{0} t\right)+E_{2} g(t-\tau) \exp \left(-i 2 \omega_{0} t\right. \\
& +i \Phi)+ \text { c.c., }
\end{aligned}
$$

where $E_{i} g(t)$ describes a slowly varying envelope with amplitude $E_{i}(i=1,2)$. With the so-called time-shifting identify $g(t-\tau) \Leftrightarrow G(\Omega) \exp (i \Omega r)$, where $g(t) \Leftrightarrow G(\Omega)$ is the Fourier transformation, the measured spectrum at the detector is given by

$$
\begin{gathered}
I\left(2 \omega_{0}+\Omega\right) \propto|G(\Omega)|^{2}\left[\left(E_{1}^{2}+E_{2}^{2}+2 \alpha E_{1} E_{2} \cos \left(2 \omega_{0} \tau\right.\right.\right. \\
+\Omega \tau+\Phi)],
\end{gathered}
$$

where $\Omega$ denotes the deviation of the frequency from the central frequency $2 \omega_{0}$ within the spectrum of SHG output. The second term in the cosine $\Omega \tau$ leads to beating in the spectrum of the SH light. The phase of the beats is directly related to the phase $\Phi$ of the response from the sample. Thus the phase information can be easily recovered just by use of a spectrometer.

In the experimental demonstration the phase of the MSHG response from a $\mathrm{Rh}-\mathrm{Co}-\mathrm{Cu}$ multilayer was measured. In the longitudinal geometry the s-polarized SHG from this isotropic sample should be odd in $\mathbf{M}$ (see Eq. 4) so that reversal of the magnetization must change the phase of the response by $180^{\circ} .47$ A 3 mm-thick glass plate was used to introduce the time delay $\tau$ between the SHG response from the sample and the reference (a thin, poled polymer film with a high second-order nonlinearity); see Fig. 7(a). The beating part of the SHG spectrum is shown in Fig. 7(b) for opposite directions of $M$. The phase change introduced by the magnetization reversal is found to be $\Phi(-M)-\Phi(+M)=176^{\circ} \pm 5^{\circ}$, in excellent agreement with the expected $\pi$ shift.

In Subsection 4.A we will describe the application of this technique to the surface MSHG from Ni(110).

\section{MSHG FROM MAGNETIC SURFACES}

A clean, magnetic surface is the benchmark object for a nonlinear MO experiment. From the theoretical point of view, it is also the simplest one. The experimental study of it, however, requires utmost care, mostly devoted to the preparation of a well-defined and clean surface.

\section{A. Ni(110) Surface: Spin-Dependent Spectroscopy}

The spin-dependent electronic structure of ferromagnetic surfaces and interfaces forms the fundamental basis for understanding surface magnetic phenomena. The following example (taken from Ref, 23) shows how MSHG can be used to study the electronic surface states on a ferromagnetic metal surface.

Experiments were performed at room temperature on a disk-shaped, Ni(110), single crystal placed in ultrahighvacuum conditions between the poles of an electromagnet. In a standard procedure, the sample surface was cleaned by repeated cycles of $\mathrm{Ar}^{+}$sputtering and annealing until no contamination could be traced. The MSHG experiments started with observation of the influence of $\mathrm{O}_{2}$ absorption. $\mathrm{O}_{2}$ exposure was done at approximately 3 $\times 10^{-9}$ mbar. With Auger electron spectroscopy, the coverage of the sample with oxygen was found to be a nonlinear function of exposure that saturated at $45 \mathrm{~L}$. Such saturation coverage corresponds to $2 \mathrm{ML}$ of $\mathrm{NiO}$.

Figure 8 shows the $\mathrm{O}_{2}$-exposure dependence of the MSHG signal in the $p_{\text {in }} p_{\text {out }}$ polarization combination. It can be seen that when the clean surface is exposed the SHG intensity increases and has one maximum at $1 \mathrm{~L}$ and another at $\approx 10 \mathrm{~L}$. Close to $20 \mathrm{~L}$, the intensity drops by more than an order of magnitude, with further increase to saturate at $\approx 45 \mathrm{~L}$. The two maxima observed seem to be well in line with the appearance of the $c(2$ $\times 1)$ and $c(3 \times 1) \mathrm{O}_{2}$-induced surface superstructures. These superstructures could be observed, at these same 
coverages, by low-energy electron diffraction. The magnetic asymmetry plotted in Fig. 8 roughly indicates the changes in the ratio $\chi^{\mathrm{magn}} / \chi^{\mathrm{cr}}$. Clearly, very small amounts of $\mathrm{O}_{2}$ drastically change the asymmetry, including a change of sign. On the other hand, the observed SHG intensity maxima at $1 \mathrm{~L}$ and $10 \mathrm{~L}$ do not visibly correlate with the MSHG asymmetry at all.

Spectroscopic MSHG has been carried out on the same surface with an aim of obtaining a better insight into the origin of the nonlinear MO response. A tunable optical parametric amplifier pumped by a Ti:sapphire regenerative amplifier was used to produce the fundamental light pulses in the wavelength range $750-1000 \mathrm{~nm}$. 'To normalize the measured SH intensity from the sample, the SHG intensity from a $c$-cut quartz crystal in the transmission geometry was measured with a second photomulti-

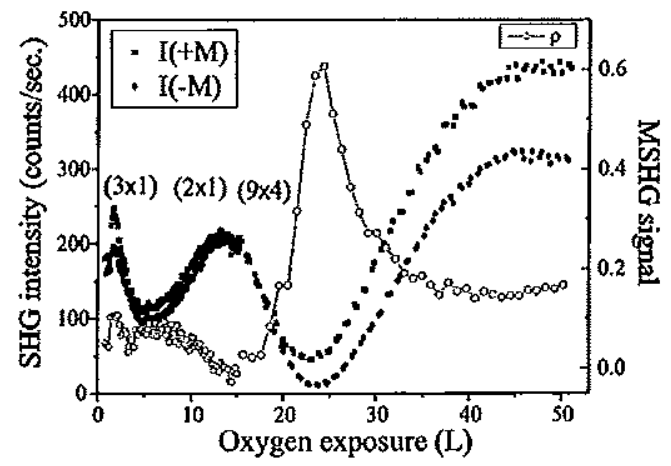

Fig. 8. SHG intensity from Ni(110) surface as a function of oxygen dose for opposite directions of $\mathbf{M}$, as well as the MSHG asymmetry.
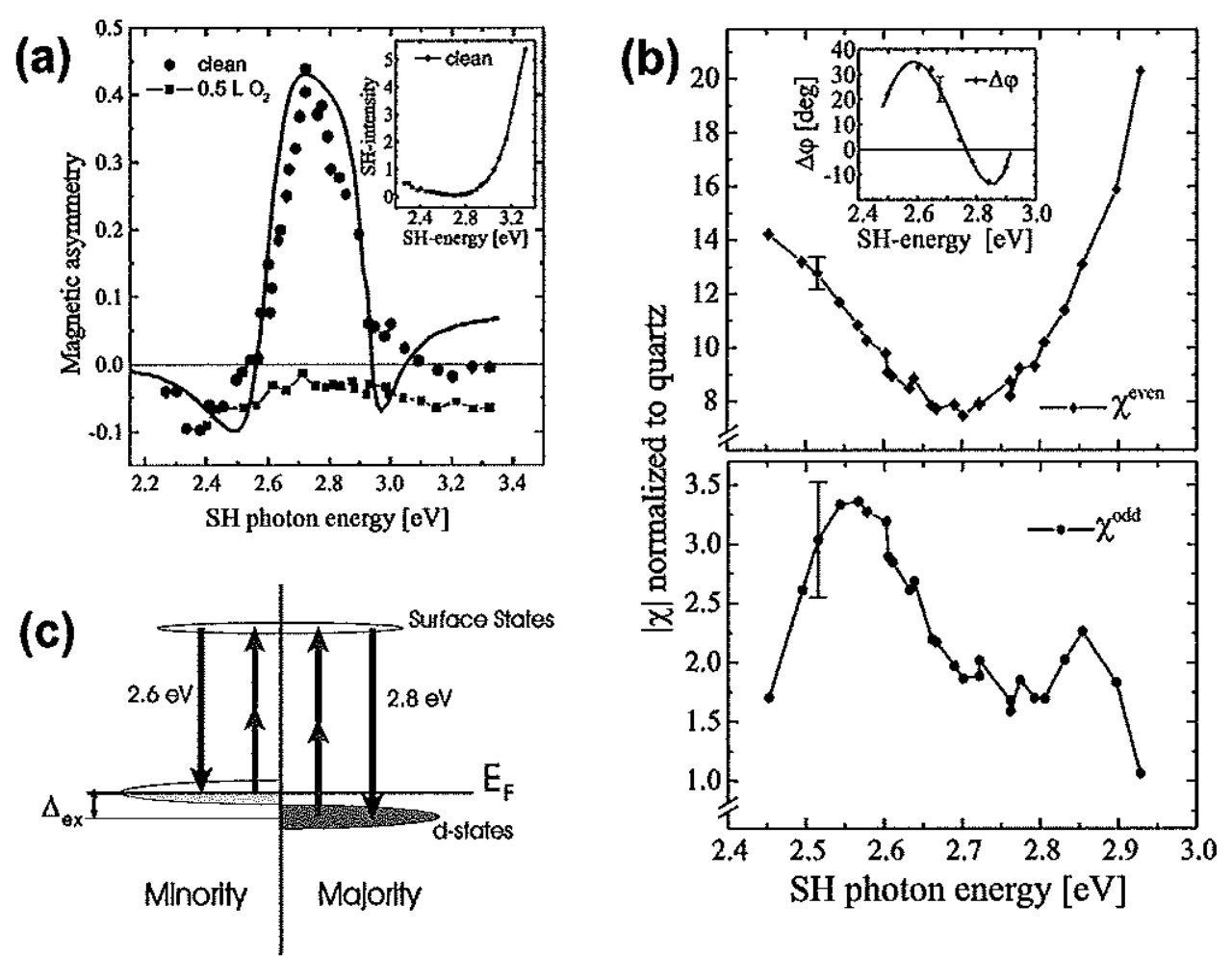

Fig. 9. (a) MSHG asymmetry and total intensity (see inset) as a function of $2 \omega$ photon energy as measured on a clean and oxidized Ni(110) surface. (b) Amplitude of the effective tensor elements as derived from the measured intensity, asymmetry, and relative phase (inset). (c) Schematic picture of the exchange split density of states and empty surface states in nickel. Reproduced from Ref. 23. plier tube. The phase of the SHG was measured by the ultrahigh-vacuum-compatible-phase-sensitive-detection technique (see Subsection 3.B above).

The magnetic asymmetry measured in the $p_{\text {in }} p_{\text {out }}$ polarization combination is plotted in Fig. 9(a) as a function of $2 \hbar \omega$ photon energy. The open circles represent the response of a clean surface while the filled squares are the response of a surface very slightly contaminated with $\mathrm{O}_{2}$ $(0.5 \mathrm{~L})$. In the inset, the average SHG intensity measured on a clean surface is shown. The magnetic asymmetry has a sharp maximum at $2.7 \mathrm{eV}$ and changes sign at 2.6 and $3.1 \mathrm{eV}$. This resonant feature completely disappears upon oxidation, clearly proving its surfacespecific nature. The relation between the effective susceptibilities and the intensity data of Fig. 8 is given by

$$
4\left|\chi_{\mathrm{eff}}\right|^{2}=I(+M)+I(-M) \pm 2[I(+M) I(-M)]^{1 / 2} \cos \Delta \phi
$$

where $\Delta \phi$ is the phase difference between the SH fields $E(2 \omega,+\mathbf{M})$ and $E(2 \omega,-\mathbf{M})$. This phase difference has been measured as a function of frequency and is shown in Fig. 9(b), along with the resulting effective susceptibilities $\left|\chi_{\text {eff }}^{\text {odd }}\right|$ and $\left|\chi_{\text {eff }}^{\text {even }}\right|$. What is surprising, the sharp maximum of Fig. 9(a) corresponds to the two-peak structure of $\left|\chi_{\mathrm{eff}}^{\text {odd }}\right|$ combined with a minimum of $\left|\chi_{\mathrm{eff}}^{\text {even }}\right|$. tra of Fig. 9(b) can be calculated with Eq. (6) within a simple model shown in Fig. 9(c). The model involves the an empty surface state $\approx 2.5 \mathrm{eV}$ above $E_{F}$. The exchange splitting of the $d$ band leads to a maximum density of
The resonances as observed in the nonlinear MO specspin splitting of the $d$ bands around the Fermi energy and 
states for minority-spin electrons at the Fermi energy and a maximum for majority-spin electrons $\approx 250 \mathrm{meV}$ below $E_{F}$. As this surface state is of nearly pure $p_{z}$ character, the exchange splitting of the state is much smaller than the splitting of the $d$ states and can be neglected. If we include only these $d$ states and surface states in the summation of Eq. (6), $\left|\chi_{\mathrm{eff}}^{\text {odd }}\right|$ can be written as

$$
\chi^{\text {odd }}=A_{0}+\frac{A_{1}}{2 \hbar \omega-E_{1}+i \hbar \Gamma_{1}}+\frac{A_{2}}{2 \hbar \omega-E_{2}+i \hbar \Gamma_{2}},
$$

where the second term on the right-hand side describes the transitions of the minority-spin electrons from filled to empty states having energy difference $E_{1}$, and the third term includes the transitions of the majority-spin electrons. Because of the spin dependence of the resonant contributions to the odd tensor component, the matrix elements $A_{1}$ and $A_{2}$ should have an opposite sign and be of approximately equal amplitude. With these relations, the model can be used to fit $\left|\chi_{\text {eff }}^{\text {odd }}\right|$ to give $E_{1}$ $=2.58 \mathrm{eV}$ and $E_{2}=2.85 \mathrm{eV}$. With $\left|\chi_{\text {eff }}^{\text {even }}\right|$ from Fig. $9(\mathrm{~b})$, it is now possible to calculate the magnetic asymmetry shown in Fig. 9(a) by the solid curve. The typical features of the asymmetry such as the two sign changes and the maximum are described very well by the model. This proves that these features do indeed arise as a result of the difference in exchange splitting between the initial $d$ states and the final surface states, which proves once again that MSHG spectroscopy can be a powerful tool to probe the spin-dependent electronic structure of surfaces.

\section{B. Magnetic Moments of Co During Growth on Cu Surface}

The following experiment made direct use of the enormous sensitivity of MSHG to the surface magnetism as well as to structure and morphology. Jin et al. ${ }^{21}$ studied in situ the layer-by-layer growth of Co films on $\mathrm{Cu}(001)$. During the growth of the Co film (very slow growth with a rate of about $7 \mathrm{ML} / \mathrm{h}$ ) MSHG signals were measured in the transverse MO geometry.

Figure 10(a) shows the total SHG intensity for $s$-polarized incident light as a function of the Co film thickness $d_{C_{0}}$ for the two opposite magnetization directions. The SHG yield from the uncovered $\mathrm{Cu}$ surface is much smaller than that from a Co film. Therefore, at the beginning of the Co deposition the SHG intensity increases almost linearly. The onset of a difference in the $I(+M)$ and $I(-M)$ at $d_{\mathrm{Co}}=1.5 \mathrm{ML}$ indicates the onset of magnetic order. The corresponding magnetic asymmetry is shown in Fig. 10(b) and the average intensity in Fig. 10(c). On top of the overall thickness dependence, an oscillation with a 1-monolayer period is clearly visible up to $d_{\mathrm{C}_{0}}=7$ to $8 \mathrm{ML}$. The slowly varying part is caused by changes in the electronic structure with increasing film thickness and the appearance of quantum-size effects in the SHG.

However, the 1-ML-period oscillations must rather be related to the morphology of the surface than to the electronic structure. It is well known that $\mathrm{SHG}$ is quite sensitive to surface morphology. For example, on a stepped Al surface the intensity changes by almost two orders of magnitude depending on the step density and step orientation. $^{68}$ The enhancement of SHG from this atomic-scale roughness is caused by the modified electronic structure of the flat surface. Co grows on $\mathrm{Cu}$ in a nearly layer-by-layer growth mode. ${ }^{69}$ Periodically, Co islands nucleate, grow in size, and coalesce (see Fig. 11), thus causing the total length of step edges to oscillate. Therefore, the observed oscillatory component in the SH intensity may be attributed to the oscillationally varying step density.

The same periodicity is seen not only in the intensity, but in the asymmetry as well. It is very well revealed in the $s_{\text {in }} p_{\text {out }}$ polarization combination (see Fig. 10), where the effect of morphology on the average SHG intensity is smaller compared with the $p_{\text {in }} p_{\text {out }}$ one. ${ }^{21}$ For clarity, the rapidly varying component of the asymmetry is calculated as the difference between $A$ and its smoothly varying course.

A difficult point in the data treatment was to decide whether the increase of the measured asymmetry corresponds with an increase of the surface magnetization or with its decrease. This could be solved by a continuous increase of the film thickness up to $150 \mathrm{ML}$, so that the contribution of the buried interface vanished. It was

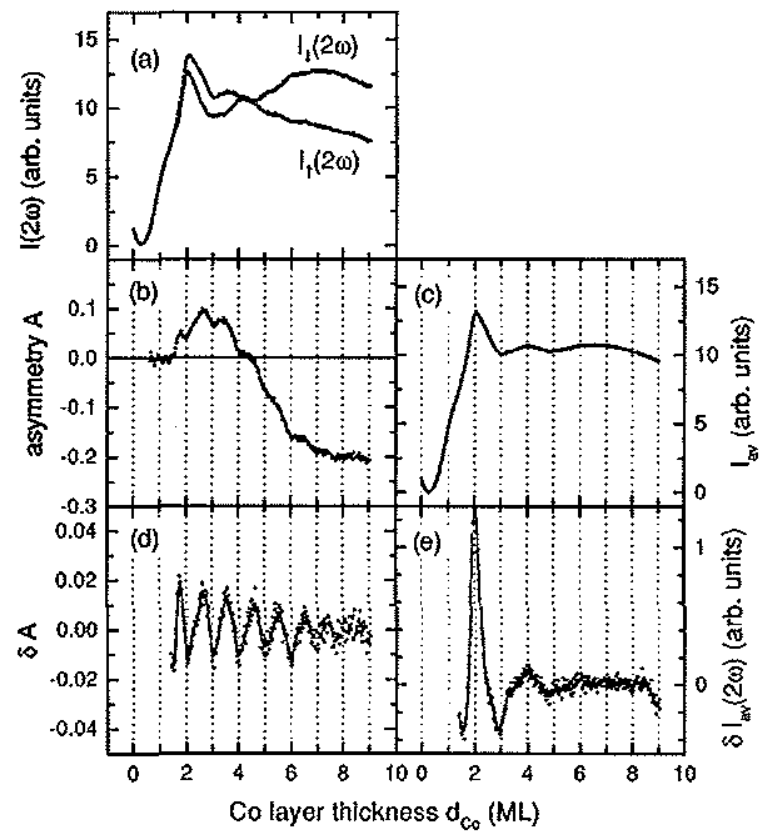

Fig. 10. (a) Measured total SHG intensities as a function of the Co film thiekness for s-polarized incident light in transverse geometry. (b) Magnetic asymmetry and (c) average SHG intensity calculated from data of (a). In (d) the difference of the magnetic asymmetry and its running average over 50 data points is plotted. (e) Similar to (d) but for the average SHG intensity. Reproduced from Ref. 21.

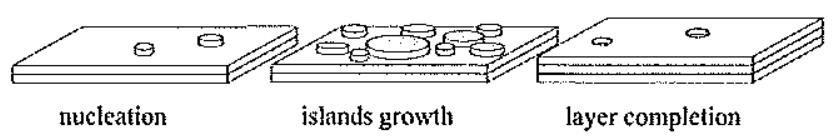

Fig. 11. Layer-by-layer growth of a thin film: appearance, growth, and coalescence of islands periodically change the number of step atoms. 

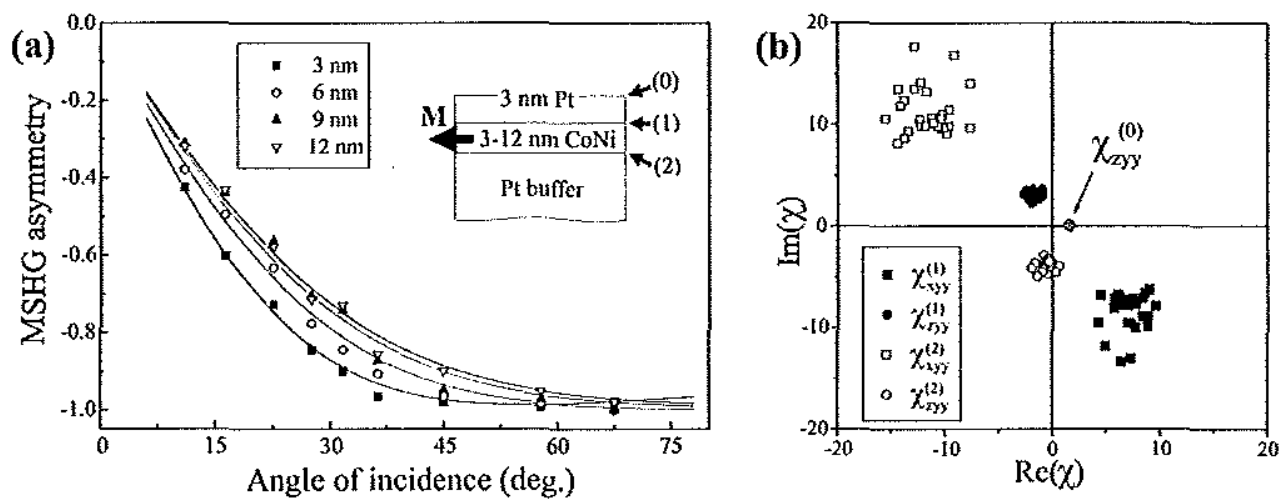

Fig. 12. (a) Angle of incidence dependences of the MSHG asymmetry for $\mathrm{Pt}-\mathrm{CoNi}-\mathrm{Pt}$ samples with different CoNi layer thicknesses (indicated in the figure). Curves are the theoretical fit to the experimental points with one single set of tensor elements. (b) The nonlinear tensor components derived from the fits in (a). Reproduced from Ref. 24.

thus shown that the increase of the magnetizationinduced asymmetry at half-filled layers indeed comes from the enhancement of the magnetic surface nonlinearity in the whole thickness range.

Though MSHG, as with the linear MOKE, does not measure the magnetization directly, it has been shown by Pustogowa et $a l .^{30}$ that the magnetic tensor elements of the second-order susceptibility depend linearly on the magnetization to a first approximation. Provided the amplitude of the change is small, variations of the asymmetry relate directly to the variations of the tensor elements. Therefore the observed increase of the asymmetry at half-filled layers suggests an increase of the magnetic moments of the atoms at step sites as is expected from the simple argument of reduced coordination number at these sites. It is not easy by any means to estimate the absolute increase of the step magnetic moments, as the probing depth of MSHG is not known exactly. Very roughly, the experimental data of Jin et al. fall in line with theoretical estimates of an increase in moment of a few percent at the step edges.

These measurements have proved the ability of MSHG to detect changes in the magnetic moment at interfaces of the order of $1 / 50 \mu_{B}$ per atom.

\section{INTERFACES IN MAGNETIC MULTILAYERS}

A. Investigation of Buried Interfaces of CoNi-Pt Films with MSHG

This section describes the first successful attempt (from Ref. 24) to derive the relative, nonlinear, MO susceptibilities per interface from experimental MSHG data.

The samples for this study were $\mathrm{Pt}-\mathrm{CoNi}-\mathrm{Pt}$ sandwiched layers prepared in a computer-controlled sputtering system with base pressure of $5 \times 10^{-8} \mathrm{mbar}$ and with Ar as a sputtering gas. The deposition rates were kept low $(0.17-0.2 \mathrm{~nm} / \mathrm{s}$ for Pt and $0.04-0.06 \mathrm{~nm} / \mathrm{s}$ for $\mathrm{CoNi})$ to assure a smooth layer: growth and a good control of layer thickness. A 40-nm-thick Pt buffer layer was deposited on a $\mathrm{Si}(001)$ substrate followed by a magnetic CoNi layer (thickness varied between 3 and $12 \mathrm{~nm}$ ) and covered by a 3-nm-thick Pt cap layer. Such samples were prepared at different Ar pressures (between 4 and $36 \mu$ bar). It was found that the magnetic properties of the samples depend considerably on the growth conditions, in particular on the Ar pressure used for sputtering.

Experimentally, the asymmetry of the MSHG signal $A$ as defined by Eq. (21) was measured as a function of the angle of incidence.

To determine the $\chi^{(2)}$ tensor for one given interface quality, a set of samples was used with different magnetic layer thicknesses prepared under exactly the same conditions (including of course the sputtering pressure of $p_{\mathrm{Ar}}$ $=12 \mu \mathrm{bar})$. It was therefore assumed that the $\chi^{(2)}$ are the same for the different samples, and the only things that are changed are the local optical fields at the interfaces due to absorption and multiple scattering. To fit the data, the transfer-matrix technique described in Subsection 2.C was employed with nonlinear susceptibilities as fitting parameters.

The results of the measurements together with the fitting curves are shown in Fig. 12(a) for the $s_{\text {in }} p_{\text {out }}$ polarization combination. The number of fitting parameters is determined by the polarization used. Thus for $s_{\text {in }} p_{\text {out }}$ one can arbitrarily fix the only tensor component of the cover layer surface $z y y_{0}$ (neither the absolute intensity nor the optical phase of MSHG is taken into account), hence $z y y_{1,2}$ and $x y y_{1,2}$ are the only components left to be determined linset in Fig. 12(a) shows the indexing of the interfaces). This leaves eight parameters (two interfaces $\times$ two complex components) to describe these data fully. The uniqueness of the fits was checked for both $s_{\text {in }} p_{\text {out }}$ and $p_{\text {in }} p_{\text {out }}$ polarization combinations by randomizing the initial choice of the fit parameters. Figure 12(b) shows the $\chi^{(2)}$ tensor components obtained from the fits of Fig. 12(a). The convergence of the parameters is evident. An interesting point is that the tensor components show opposite signs for the subsequent magnetic layer interfaces (1) and (2). This is independent experimental confirmation of a strict requirement from symmetry ${ }^{70}$ and provides a strong support for the model used in the calculations. Also the crystallographic and magnetic contributions to $\chi^{(2)}$ appear to be of the same order of magnitude, in strong contrast with the linear case.

To determine the dependence of $\chi^{(2)}$ on the interface quality, the sample with a 3-nm-thick CoNi layer was measured for different Ar sputtering pressures. The as- 
sumption was then made that all tensor components changed in a similar way, i.e., scaling parameters $\mathcal{M}$ and $\mathcal{C}$ could be defined as

$$
\begin{aligned}
\chi_{\text {magn }}^{(2)}\left(p_{\mathrm{Ar}}\right) & =\mathcal{M}\left(p_{\mathrm{Ar}}\right) \cdot \chi_{\text {magn }}^{(2)}\left(p_{0}\right), \\
\chi_{\mathrm{cr}}^{(2)}\left(p_{\mathrm{Ar}}\right) & =\mathcal{C}\left(p_{\mathrm{Ar}}\right) \cdot \chi_{\mathrm{cr}}^{(2)}\left(p_{0}\right),
\end{aligned}
$$

with $p_{0}=12 \mu \mathrm{bar}$. To fit the data for any new sample, only the two complex parameters $\mathcal{M}$ and $\mathcal{C}$ are used (actually this gives only three parameters in total because one phase can still be fixed). The possibility of fitting the data for any Ar pressure in such a manner supports the assumption that all $\chi^{(2)}$ are changed in a similar way.

The parameters $\mathcal{M}\left(p_{\mathrm{Ar}}\right)$ and $\mathcal{C}\left(p_{\mathrm{Ar}}\right)$ represent the dependence of the nonlinear MO interface properties on the interface structure (controlled through sample preparation conditions). The value of $\mathcal{C}$ is proportional to the crystallographic contribution to the MSHG, expressed through the local symmetry breaking induced by the interface. It is incorrect to say, however, that $\mathcal{M}$ represents the purely magnetic part of MSHG. Indeed, all the "magnetic" elements of $\chi^{(2)}$ are nonzero only in the presence of crystallographic symmetry breaking, i.e., the same factor influences both $\chi_{\mathrm{cr}}$ and $\chi_{\text {magn }}$. Hence one may write $\mathcal{M} \propto \mathcal{C} \cdot \mathbf{M}$

To extract information on the interface magnetic properties, we take the ratio $\mathbf{m}=\mathcal{M} / \mathcal{C}$. In Fig. $13 \mathrm{~m}$ is plotted as a function of the sputtering Ar pressure for the $s_{\text {in }} p_{\text {out }}$ and $p_{\text {in }} p_{\text {out }}$ polarizations. The precise coincidence of the $\mathbf{m}$ dependency for both polarization combinations once again supports the model used for the derivations.

Figure 13 shows that the crystallographic contribution $\chi_{\mathrm{cr}} \propto \mathcal{C}$ increases rapidly above $15 \mu$ bar after staying almost constant below this pressure. The increase of $\chi_{\mathrm{cr}}$ indicates an increasing interfacial roughness for higher sputtering pressures. Though the crystallite size is known to stay constant in the whole pressure range, the crystallites may become slightly misoriented. ${ }^{64}$ This increase of $\chi_{\text {cr }}$ due to increasing interface roughness can be understood schematically as being due to the increase of the effective surface area of the interface. For stronger roughness, other mechanisms may play a role. ${ }^{71}$

In contrast to the crystallographic case the magnetic contribution $m$ shows a clear maximum at pressures of 15-20 $\mu$ bar. At very low Ar pressures the interface layers become slightly intermixed due to the high energies of sputtered atoms. This intermixing hardly affects the

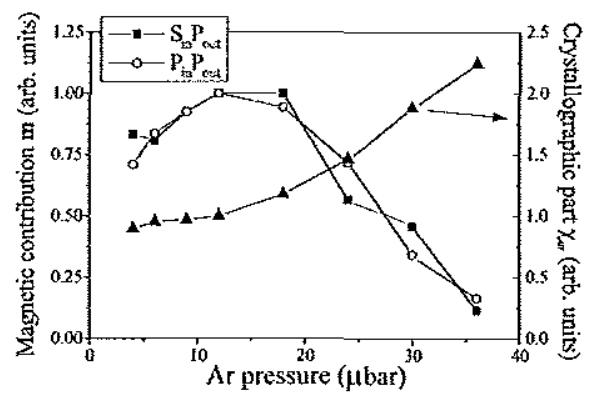

Fig. 13. Crystallographic as well as magnetic contributions to the nonlinear magneto-optical tensor derived from the two different polarization combination data (indicated in the figure). Reproduced from Ref. 24 .

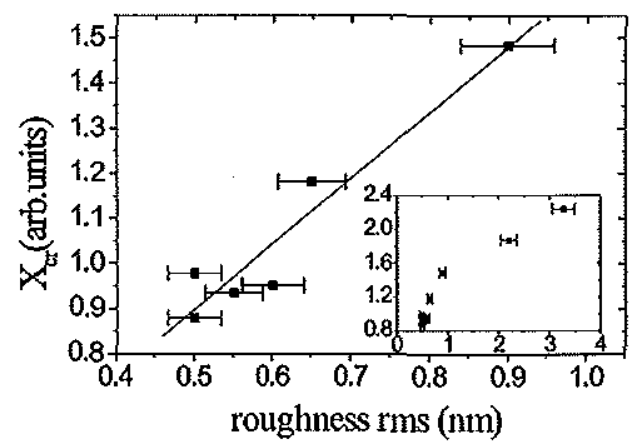

Fig. 14. Crystallographic $\chi^{(2)}$ contribution as a function of interface roughness determined from atomic force microscopy and X-ray scattering experiments. Reproduced from Ref. 72 .

crystallographic part of MSHG but clearly suppresses the magnetic one. Note that the maximum in the interface magnetization does not have to coincide with the sharpest interface. Evidently the drop of $\mathbf{m}$ for large $p_{\mathrm{Ar}}$ is related to a decreasing in-plane magnetic moment of the rough interface. A possible explanation here is that the increasing roughness changes the local coordination of the Co atoms, which may even lead to an out-of-plane lifting of the local interface magnetic moments. This explanation is supported by the observation of a specular $s$-polarized MSHG output at higher Ar pressures. ${ }^{72}$ Such an MSHG yield can only be nonzero in the presence of a perpendicular (out-of-plane) magnetization component. In addition, polar MOKE hysteresis loops also showed a small remanence ( $\leqslant 10 \%$ of $M_{s}$ ) for the sample sputtered at $P_{\text {Ar }}=36 \mu \mathrm{bar}$, confirming the MSHG results.

It was also determined that the value of $\chi^{\mathrm{cr}}$ is roughly proportional to the surface-interface roughness measured by other methods, such as atomic-force microscopy and grazing-incidence X-ray scattering (see Fig. 14).

To summarize, nonlinear MO is clearly able to follow (subtle) changes in interface structure, both crystallographic and magnetic. For the case of Pt-CoNi-Pt optimum sputtering pressure was found that yielded a maximum in-plane interface magnetization with only a small change in interface morphology. With further increase of the sputtering pressure, the interface roughness clearly increases while the in-plane interface magnetic moment decreases. It should be emphasized that for all samples studied, no difference in the total layer magnetization was observed with either MOKE or vibrating-sample magnetometer.

B. Interface Versus Bulk MSHG in Fe-Au Multilayers In a study of interface versus bulk the MSHG technique was applied to $\mathrm{Fe}-\mathrm{Au}$ superlattices with atomically controlled epitaxial layers. A superlattice with a modulation of monatomic layers of $\mathrm{Fe}$ and $\mathrm{Au}$ has been known to show an artificial order with an $L 1_{0}$ structure that does not exist in nature. ${ }^{73}$ Such an artificial structure remains at interfaces between $\mathrm{Fe}$ and Au layers when the modulation period becomes longer than monatomic. ${ }^{74}$ The linear MO spectra of the superlattices modulated by integer and noninteger numbers of atomic layers have been studied intensively with the results suggesting the formation of a peculiar band structure in such an artificial real-space structure. ${ }^{75,76}$ 
Figure 15 shows the results of rotational anisotropy measurements for all four polarization combinations in a longitudinal geometry. A sample with lattice period $x$ $=15 \mathrm{ML}$ was used. All curves show a clear fourfold anisotropy and a clear magnetic contrast.

In Fig. 16, the results of all four polarization combinations for the sample with $x=15 \mathrm{ML}$ are plotted for the case of transverse geometry. Note the different vertical scales for the various data, indicating a substantial difference for the MSHG response for different polarization combinations. It is also obvious that all data involving $s_{\mathrm{in}} s_{\text {out }}$ polarization yield a much stronger anisotropy, which is a direct consequence of the in-plane $x y$ tensor components that contribute to these signals (see below) Even the weakest $s_{\text {in }} s_{\text {out }}$ curve shows a clear fourfold symmetry pattern.

Usually an analysis of MSHG results ${ }^{12,13,18}$ is performed under the assumption that the top surface and buried interfaces are the only sources of the nonlinear MO response. Their nonlinearity is described in terms of the effective surface-interface, dipolelike, nonlinear susceptibility $\chi^{(2)}(\mathbf{M})$, which is a third-rank tensox: Such contribution yields the azimuthal patterns described by Eqs. (14). Note that these patterns do not yield any effect of magnetization reversal for the $s_{\text {in }} s_{\text {out }}$ and $p_{\text {in }} s_{\text {out }}$ MSHG intensity, in contrast to the experimental observations shown in Figs. 15 and 16 . They are also unable to describe properly the patterns for the other two polarization combinations (see Fig. 15 for dotted lines on the plots for the $p_{\text {in }} p_{\text {out }}$ and $s_{\text {in }} p_{\text {out }}$ polarization combinations; also in this case, the equations are not able to fit the magnetic contrast because of a different symmetry pattern; see below). Therefore one has to take into account additional anisotropic contributions to the second-order nonlinear response. In particular the nonlocal (quadrupoleallowed) contribution from the bulk of cubic nonmagnetic

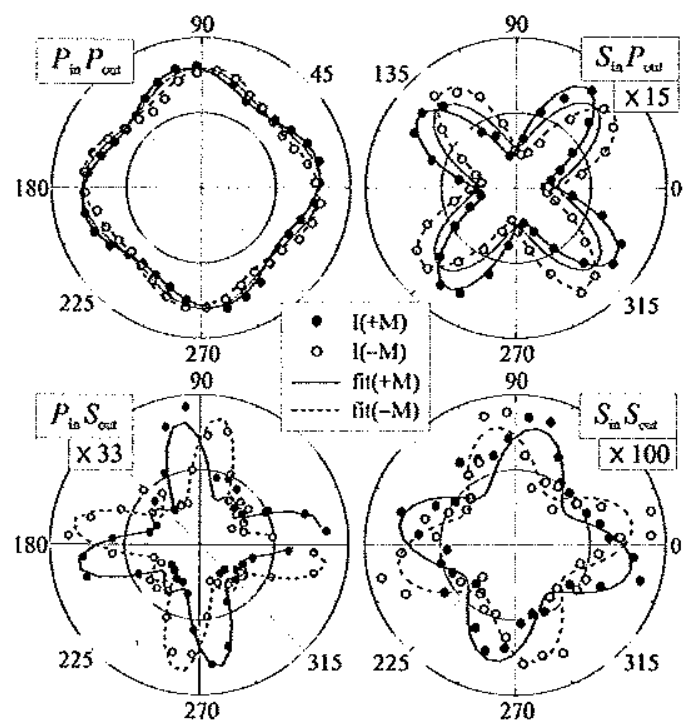

Fig. 15. Rotational anisotropy curves (experimental points plus theoretical fits) for the sample with single layer thickness $x$ $=15$ monolayers in longitudinal geometry. Multiplication factors in three plots show the scaling of the corresponding data in order to reach the same intensity level as with the $p_{\text {in }} p_{\text {out }}$ polarization combination (this figure and Fig. 16). Reproduced from Ref. 45.

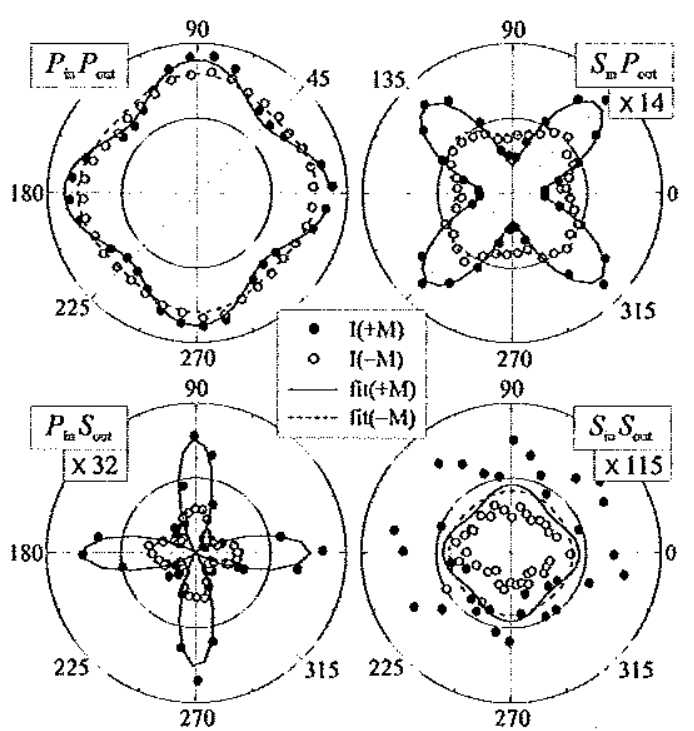

Fig. 16. Rotational anisotropy curves for the sample with single layer thickness $x=15$ monolayers in transverse geometry. Reproduced from Ref. 45 .

metals $\left(\mathrm{Cu}^{77} \mathrm{Ag}^{78}\right.$ and $\left.\mathrm{Al}^{79}\right)$ and semiconductors $\left(\mathrm{Si}^{80}\right)$ has been shown to lead to a fourfold anisotropy of SHG at their (100) surfaces. Accounting for this additional contribution modifies the rotational patterns to those described by Eqs. (18). Use of these equations for the theoretical fits to the experimental data of Figs. 15 and 16 resulted in good agreement between experiment and theory.

Thus we have shown that the MSHG response of Fe$\mathrm{Au}(001)$ superlattices shows a strong azimuthal anisotropy in both the MSHG intensity and in the nonlinear MOKE rotation. These observations can be fully described by taking into account not only the interfaceallowed dipole contributions but the higher-order (bulklike) quadrupole contributions. This result is fully consistent with observations from other nonmagnetic (001) surfaces but was not evident a priori because, in principle, a dipole-allowed magnetic contribution alone could provide the necessary anisotropy. Moreover, independent of the details of the nonlinear optical response, very general and powerful statements can be made that relate the observed MSHG response to the magnetic and crystallographic symmetries. This is in line with similar observations that were recently made by Fiebig $e t a l$. when they used MSHG to solve the symmetry of the spin ordering in several antiferromagnetically ordered crystals ${ }^{81}$ that could not be solved by neutron scattering. This shows once more that MSHG is indeed a powerful tool in revealing the erystallographic and magnetic symmetry of spin-ordered systems.

\section{G. Magnetization Reversal at the Interfaces}

In the discussion of thin magnetic films and multilayers, interfaces play an often dominant role in determining magnetization reversal behavior. To determine the behavior of the magnetization at the film interfaces as opposed to that in the middle of the film, MSHG can be used in combination with the linear MOKE technique. The magnetization-reversal hysteresis is thus measured si- 
multaneously from the same spot on the sample. ${ }^{82,83}$ Figure 17 shows an example of such experiment in amorphous TbFeCo layers that have recently attracted attention as possible MO hybrid recording media. ${ }^{81}$ MOKE and MSHG hysteresis loops were measured for different sample temperatures across the Curie point $T_{\mathrm{C}}$ $=465 \mathrm{~K}$. A clear difference between the results of the two methods was observed that can be related only to the different magnetic behavior of the bulk and of the interfaces of the film. Closer inspection of the loops in Fig. 17 seems to indicate that the reversal starts at the interfaces but somehow stays pinned in the bulk, thus slowing down the bulk reversal. ${ }^{82}$

\section{EXTENSIONS OF NONLINEAR MAGNETO-OPTICS}

\section{A. Plasmon-Amplified MSHG}

Surface collective electron oseillations, also known as surface plasmons (SPs), can be excited in noble metals below the plasma frequency and may give rise to a variety of linear and nonlinear phenomena. ${ }^{85}$ The coupling of the electric field at optical frequencies with SPs in metallic multilayer films results in an enhancement of the linear MO effects. ${ }^{86}$ It has been shown experimentally and theoretically that SHG is also strongly enhanced due to SP excitation. ${ }^{85,87}$

Here we discuss the experimental observation of nonlinear MO phenomena related to SP excitation in an ultrathin Au-Co-Au multilayer structure (after Ref. 88). The measurements have been made by the attenuatedtotal-reflection technique in the Kretschmann geometry. ${ }^{85,88}$

The ultrathin Au-Co-Au films were deposited on a 1-mm-thick float glass substrate in a high-vacuum chamber by the procedure described in Ref. 89 . In the $\mathrm{Au}(3 \mathrm{~nm})-\mathrm{Co}(3 \mathrm{~nm})-\mathrm{Au}(25 \mathrm{~nm})$-glass structure the easy magnetization axis was located in the film plane. The glass substrate was optically coupled to a half-cylindrical glass lens by a refractive-index adaptation liquid. The experimental setup and the geometry of measurements in the attenuated-total-reflection configuration are shown in the inset to Fig. 18; for further experimental details, see

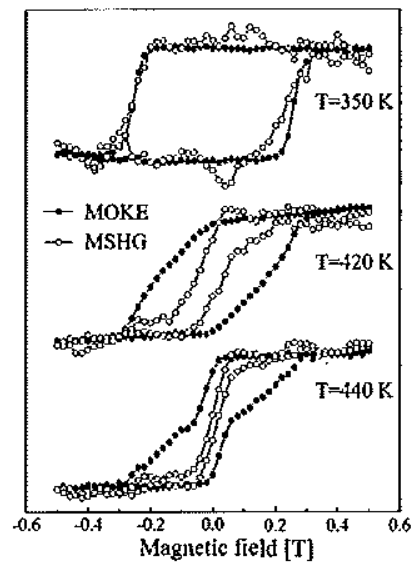

Fig. 17. MOKE and MSHG hysteresis loops from a TbFeCo MO recording layer for three different sample temperatures. Reproduced from Ref. 82.

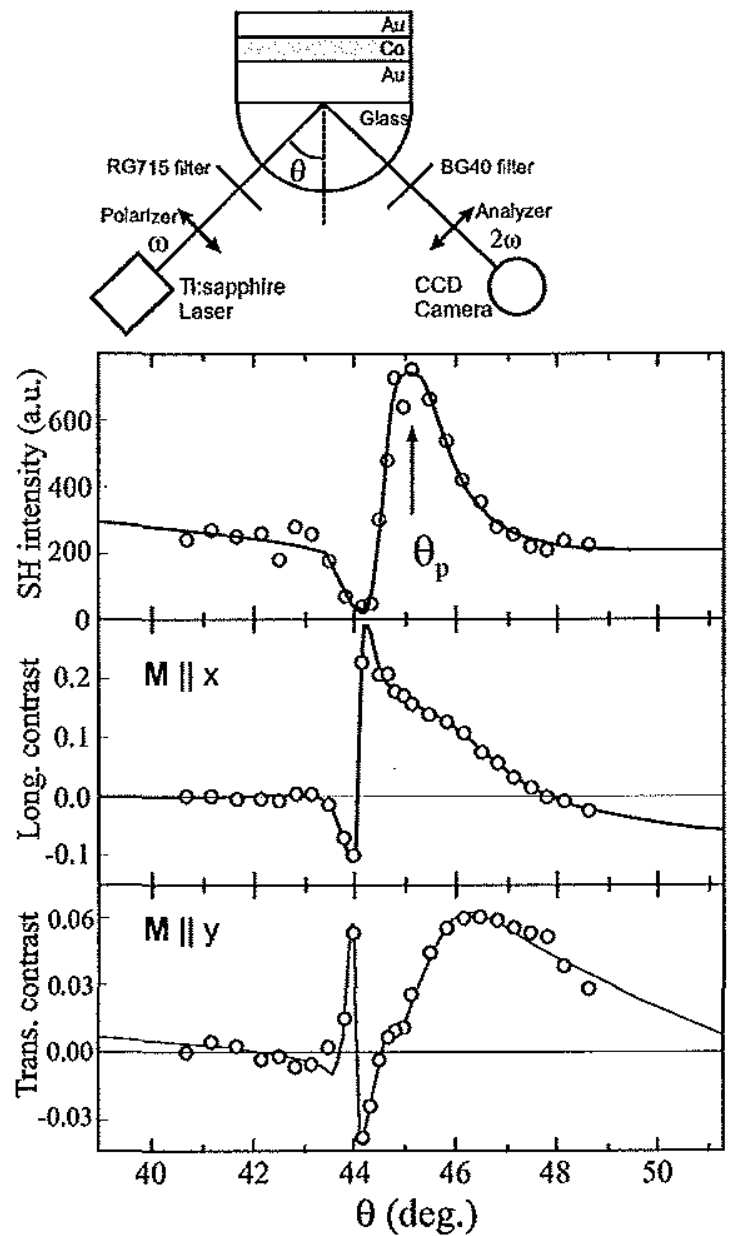

Fig. 18. MSHG as a function of the angle of incidence near the critical angle for SP excitation; sample is schematically shown at top. Reproduced from Ref. 88 .

Ref. 88. $p$-polarized fundamental light (TM mode) was used that excites SPs near an incidence angle $\theta_{p}$ with a wave vector $k_{p}$ defined by the SP dispersion equation ${ }^{85}$

$$
k_{p}=\frac{\omega}{c}\left(\frac{\epsilon_{1} \epsilon_{2}}{\epsilon_{1}+\epsilon_{2}}\right)^{1 / 2},
$$

where $\epsilon_{1}$ and $\epsilon_{2}$ are the dielectric constants of the two media at the interface where SPs are excited. SPs may be coupled with evanescent optical waves from the halfcylindrical glass lens at the angle

$$
\theta_{p}=\arcsin \left(\frac{k_{p} c}{\omega \sqrt{\epsilon}}\right)
$$

where $\epsilon$ is the dielectric constant of the glass.

The total SH intensity measured in the longitudinal geometry $(M \| x)$ with $p$-polarized input and $p$-polarized output is shown by open circles in Fig. 18. There is no magnetic contribution to the nonlinear polarization in this configuration. The SH signal has a strong minimum at the angle $\theta=44^{\circ}$. It reaches a maximum near $\theta_{p}$, decreases, and is constant for angles $\theta \geqslant 47^{\circ}$. Thus the coupling of the SPs with light at the fundamental fre- 
quency gives rise to drastic changes of SH intensity. The SH magnetic contrast exhibits a sign reversal when the SPs are excited.

To explain these results a model was developed based on multiple interference of the different interface contributions to SHG, which can be calculated using the Green's-function technique. The Green's-function approach was used for general description of SHG from surfaces and interfaces by Guyot-Sionnest et al. ${ }^{90}$ and for analysis of the SHG dispersion at simple metal surfaces by Liebsch and Schaich. ${ }^{91}$ The Green's-function formalism is a convenient way for consideration of the light propagation problem in multilayer structures when the layer thickness is much less than the light wavelength. In this model we took into account the coupling of SPs with the fundamental light by using a theory developed for SP excitation in trilayer metallic systems. ${ }^{92}$

In the electric-dipole approximation SHG is allowed at magnetic and nonmagnetic interfaces in these films. At the angle $\theta_{p}$ the fundamental electric field is strongly enhanced at the nonmagnetic air-Au interface and redistributed to the magnetic $\mathrm{Au}-\mathrm{Co}$ and $\mathrm{Co}-\mathrm{Au}$ interfaces as a result of the coupling of SPs with the fundamental light. This results in an enhancement of both the MSHG and the nonmagnetic SHG. Drastic changes in the distribution of the fundamental field at the magnetic interfaces near $\theta_{p}$ result in a sign reversal of the SH magnetic contrast in the longitudinal and transverse geometries.

One should mention here that the effects of SPP generation on MSHG have also been observed in nanomaterials. ${ }^{93}$

\section{B. Magnetization-Induced Sum-Frequency Generation from Surface Plasmons}

In fact SHG is just a degenerate case of the general nonlinear optical process of three-wave mixing $\omega_{1}+\omega_{2}$ $=\omega_{3}$. The argument of symmetry breaking at interfaces, which yields the interface specificity of MSHG, should apply equally to magnetization-induced-sumfrequency generation (MSFG). Although SFG has been used for surface studies for more than a decade, ${ }^{94-96}$ nothing is known of the magnetic properties of SFG. Compared with MSHG, MSFG would allow for many more spectroscopic opportunities, for example to probe magnetic excitations at surfaces and interfaces.

The nonlinear optical polarization induced in a sample by two incident optical fields can be written as

$$
P_{i}^{(2)}\left(\omega_{\mathrm{sfg}}\right)=\chi_{i j k}^{(2)}\left(\omega_{\mathrm{sfg}} ; \omega_{\mathrm{ir}}, \omega_{\mathrm{vis}}\right) E_{j}\left(\omega_{\mathrm{ir}}\right) E_{k}\left(\omega_{\mathrm{vis}}\right),
$$

where $\omega_{\mathrm{sfg}}, \omega_{\mathrm{ir}}$, and $\omega_{\text {vis }}$ are the frequencies of the SFG, infrared, and visible beams, respectively, and $\chi_{i j k}^{(2)}$ is the second-order nonlinear optical-susceptibility tensor similar to that used for the description of MSHG. Both SHG and SFG are described by a third-rank tensor that vanishes in media with inversion symmetry, yielding the sensitivity of both SHG and SFG to symmetry-breaking interfaces. In the same way one can also immediately derive the nonzero $\chi_{i j k}^{(2)}$ elements that appear in the presence of magnetization, as they are the same for MSHG and MSFG. However, although in the former case the response is determined purely by the electronic density of states, for MSFG both the electronic as well as vibronic (phonons, magnons) states can contribute to the nonlinear optical response. While this is rather a practical limitation due to the measurement techniques, it is nevertheless a key point of interest in the SFG technique in general.

The direction in which this polarization radiates is found from the conservation of momentum parallel to the interface:

$$
k_{x}\left(\omega_{\mathrm{sfg}}\right)=k_{x}\left(\omega_{\mathrm{vis}}\right)+k_{x}\left(\omega_{\text {ir }}\right) .
$$

The access to the infrared region opens the possibility of studying the interaction of surface plasmon-polariton (SPP) modes ${ }^{85}$ with magnetization. In the visible, there is always a strong interference of these modes with interband transitions. However with photon energies below a few tenths of an electron volt one can obtain not only an effective excitation but also propagation over macroscopic distances of these SPPs. Because SPP modes are confined to the interface and propagate along its plane, they are particularly sensitive to interface properties. In addition, as a result of a focusing effect, the field of an SPP can be considerably larger than the field used to excite it, leading to an enhancement of the nonlinear optical response. ${ }^{97}$

The excitation of an SPP by an incident electromagnetic wave has to involve a coupling method that takes care of the excess of momentum carried by the SPP. Here a grating with a period of $d=5 \mu \mathrm{m}$ is used for that purpose. For the effective excitation one has to choose the proper wavelength for a given angle of incidence. The wave vector of the SPP is given by

$$
K^{\mathrm{SPP}}=k_{x}\left(\omega_{\mathrm{ir}}\right) \pm k_{\text {grat }},
$$

with $k_{x}$ the component of the wave vector of the IR input radiation along the interface. For wavelengths longer than the grating period $d$ this leads to the following resonant condition (see also inset in Fig. 17):

$$
\lambda_{\text {res }}^{\mathrm{ir}}=d(1+\sin \alpha),
$$

which yields $\lambda_{\text {res }}^{\text {ir }}=8.99 \mu \mathrm{m}$ for $\alpha=53^{\circ}$.

The following experiments ${ }^{98}$ were done at the FreeElectron Laser for Infrared eXperiments (FELIX) in Nieuwegein, The Netherlands, which delivers tunable IR radiation with wavelengths in the range of $5-240 \mu \mathrm{m}$. This IR radiation comes in bursts, so-called macropulses, typically $5 \mu$ s long at a $5-\mathrm{Hz}$ repetition rate. Each macropulse contains a large number of 1-3-ps-long micropulses, with a repetition rate of $1 \mathrm{GHz}$ that is given by the modulation frequency of the electron beam. The typical micropulse energy in the range of $5-50 \mu \mathrm{m}$ is $10 \mu \mathrm{J}$.

An actively mode-locked Nd:YLF oscillator was synchronized to FELIX to produce an SFG output. For proper mode-locking operation of the laser its cavity length is matched to the rf frequency of FELIX that is applied to the mode-locker crystal by actively controlling one cavity mirror with a piezo transducer. The remaining jitter was measured to be less than $1 \mathrm{ps}$. The delay between the two lasers was then adjusted with the help of an electronic phase shifter; allowing for delay changes of 1 ns maximum. 


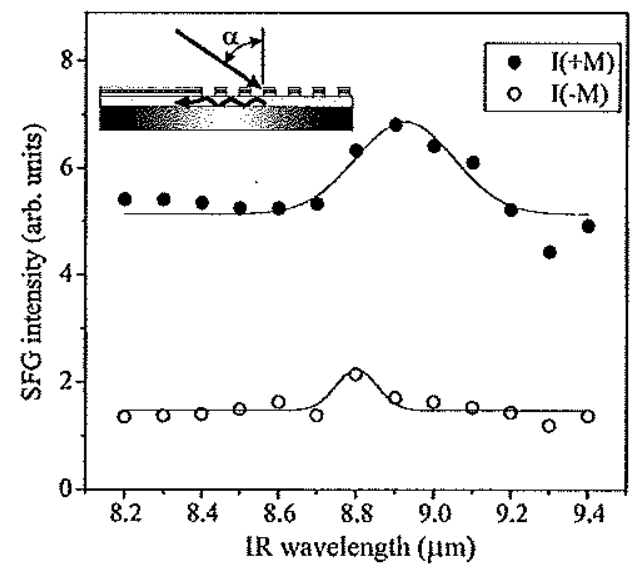

Fig. 19. SFG intensity for two opposite magnetization directions measured as a function of the infrared wavelength on a $5-\mu \mathrm{m}$-period grating made from a $\mathrm{Pt}-\mathrm{CoNi}-\mathrm{Pt}$ sandwich. Reproduced from Ref. 98 .

To obtain pulse energies comparable with those of FELIX, the output of the Nd:YLF laser is amplified several orders of magnitude, then frequency-doubled in a $\mathrm{KD}^{*} \mathrm{P}$ crystal, resulting in 7-ps pulses at $523.5 \mathrm{~nm}$ with an energy content up to $30 \mu \mathrm{J}$. The amplifier slicer was synchronized with the FELIX macropulses to obtain a similar time structure for both lasers.

Both lasers were only slightly focused (beam diameter of $\approx 1 \mathrm{~mm}$ ) onto the sample in order to stay well below the damage threshold. The angles of incidence were $45^{\circ}$ and $53^{\circ}$ for the visible and infrared beams, respectively. The sample magnetization was perpendicular to their common plane of incidence, fixed with a magnetic field.

Figure 19 shows the SFG intensity as a function of the infrared wavelength measured on the Pt-CoNi-Pt grating, where a clear resonance is observed at $\lambda=8.9 \mu \mathrm{m}$ in strict agreement with Eq. (26). The increase of the $\mathrm{SFG}$ intensity is a consequence of the buildup of the interface field due to the excitation of SPPs. The observed resonance can be fitted by the Lorentzian

$$
\chi^{(2)}(\omega)=\frac{a}{\omega_{\mathrm{SPP}}-\omega-i \Gamma}+b,
$$

where $a$ and $b$ are constants indicating the relative weights of the resonant and nonresonant contributions, $\omega_{\mathrm{SPP}}=139 \mathrm{meV}$, and $\Gamma=2.5 \mathrm{meV}$ for $I(+M)$. For the opposite magnetization direction, the very small resonant signal does not permit any numbers to be derived from the data. Figure 19 not only shows that SFG from SPPs is feasible but also that the observed resonance is strongly affected by the magnetization. This opens the way to study surface spin excitations. By changing the spatial overlap between the IR and the visible beam, the damping of the SPP and its magnetization dependence can be measured directly.

\section{CONCLUSION}

This paper updates the recent progress and milestones achieved in the newly developed area or nonlinear magneto-optics. By no means pretending to be comprehensive, it focuses on the application of MSHG to surface and interface phenomena, such as enhanced magnetic moments, electronic surface states, and correlation between interface structure and magnetism. The extreme surface-interface sensitivity of the MSHG technique manifests itself in each of the examples considered.

On the other hand, various interesting developments of MSHG are not covered in detail. Among them are the very extensive work on nonlinear MO in antiferromagnetic dielectrics, ${ }^{81,99-102}$ as well as several studies of quantum-well-state systems. ${ }^{18,61,103,104}$ In addition, one should mention the attempts to study MSHG effects in complex systems such as magnetic photonic crystals ${ }^{105,106}$ and nanoparticles ${ }^{107}$ as well as a discussion of possible MSHG effects in vacuum. ${ }^{108}$

Another area where the MSHG technique is used quite extensively is for the pump-probe studies of ultrafast magnetization dynamics. ${ }^{109-114}$ Here, MSHG has some advantages over MOKE, namely (i) because of large odd components of the nonlinear optical tensor, different components of $\mathbf{M}$ can be easily separated, and (ii) it is quite straightforward to distinguish the electron-temperature relaxation effects from those due to the transient magnetization behavior by analyzing the various tensor components. In addition the MO probe of the ultrafast magnetization dynamics can be done only with femtosecond laser pulses, which makes the measurements of the MSHG response as easy as those of the MOKE. The particular direction for which the MSHG probe can be especially interesting is the observation of magnetization dynamics at surfaces and interfaces. The spin-orbit coupling at interfaces would become accessible with this approach.

\section{ACKNOWLEDGMENTS}

The authors are very much thankful to R. V. Pisarev and $A$. Boardman who strongly encouraged them to undertake this review. The contribution of the European TMR (Training and Mobility of Researchers) network DYNAMICS (DYNAmics in MagnetIC nanoStructures), as well as that of the IST (Information Storage Technology) network SPINOSA (Spin Polarized Injection in Nanostructures and Devices), is gratefully acknowledged. Part of this work was supported by the "Stichting voor Fundamenteel Onderzoek der Materie" (FOM).

\section{REFERENCES AND NOTES}

1. Y. R. Shen, The Principles of Nonlinear Optics (Wiley, New York, 1984).

2. Nonlinear Optics in Metals, K. H. Bennemann, ed. (Clarendon Press, Oxford, UK, 1998).

3. T. F. Heinz, "Second-order nonlinear optical effects at surfaces and interfaces," in Nonlinear Surface Electromagnetic Phenomena, H.-E. Ponath and G. I. Stegeman, eds. (North-Holland, Amsterdam, 1991).

4. G. A. Reider and T. F. Heinz, "Second-order nonlinear optical effects at surfaces and interfaces: recent advances," in Photonic Probes of Surfaces, P. Halevi, ed. (Elsevier, Amsterdam, 1995).

5. A. K. Zvezdin and V. A. Kotov, Modern Magneto-optics and Magneto-optical Materials (Institute of Physics, Bristol, 1997).

6. P. S. Pershan, "Nonlinear optical properties of solids: energy considerations," Phys. Rev. 130, 919-929 (1963). 
7. E. Adler, "Nonlinear optical frequency polarization in a dielectric," Phys. Rev. 134, A728-A733 (1964).

8. J. Lajzerowicz and M. Vallade, "Génération du second harmonique dans les substances magnétiques ordonnées," C.R. Acad. Sci. Paris 264, 1819-1824 (1967).

9. N. N. Akhmediev, S. B. Borisov, A. K. Zvezdin, I. L. Lyubchanskii, and Y. V. Melikhov, "Nonlinear optical susceptibility of magnetically ordered crystals," Sov. Phys. Solid State 27, 650-652 (1985).

10. S. Kielich and R. Zawodny, "Linear and nonlinear magneto-optical effects in materials with spatialdispersion and magnetic-ordering," Physica B \& C 89, 122-125 (1977).

11. S. Kielich and R. Zawodny, "New nonlinear magnetooptical phenomena in crystals and liquids," Opt. Acta 20, 867-877 (1973).

12. R.-P. Pan, H. D. Wei, and Y. R. Shen, "Optical secondharmonic generation from magnetized surfaces," Phys. Rev. B 39, 1229-1234 (1989).

13. W. Hübner and K. H. Bennemann, "Nonlinear magnetooptical Kerr effect on a nickel surface," Phys. Rev. B 40, 5973-5979 (1989).

14. J. Reif, J. C. Zink, C. M. Schneider, and J. Kirschner, "Effects of surface magnetism on optical second harmonic generation," Phys. Rev. Lett. 67, 2878-2881 (1991).

15. J. Reif, C. Rau, and E. Matthias, "Influence of magnetism on second harmonic generation," Phys. Rev. Lett. 71, 1931-1934 (1993).

16. G. Spierings, V. Koutsos, H. A. Wierenga, M. W. J. Prins, D. Abraham, and Th. Rasing, "Optical 2nd harmonic generation study of interface magnetism," Surf. Sci. 287, 747749 (1993).

17. Th. Rasing, "Nonlinear magneto-optical probing of magnetic interfaces," Appl. Phys. B 68, 477-484 (1999).

18. H. A. Wierenga, W. de Jong, M. W. J. Prins, Th. Rasing, R. Vollmer, A. Kirilyuk, H. Schwabe, and J. Kirschner, "Interface magnetism and possible quantum well oscillations in ultrathin $\mathrm{Co} / \mathrm{Cu}$ films observed by magnetization induced second harmonic generation," Phys. Rev. Lett. 74, 14621465 (1995).

19. M. Straub, R. Vollmer, and J. Kirschner, "Surface magnetism of ultrathin $\gamma$-Fe films investigated by nonlinear magneto-optical Kerr effect," Phys. Rev. Lett. 77, 743-746 (1996).

20. R. Vollmer, M. Straub, and J. Kirschner, "Nonlinear" magneto-optical Kerr measurements on Fe(110)," Surf. Sei. 352, 937-941 (1996).

21. Q. Y. Jin, H. Regensburger, R. Vollmer, and J. Kirschner "Periodic oscillations of the surface magnetization during the growth of Co films on Cu(001)," Phys. Rev. Lett. 80, $4056-4059$ (1998).

22. K. J. Veenstra, P. E. Hansen, A. Kirilyuk, A. V. Petukhov, and Th. Rasing, "Surface magnetism of Ni(110) probed by magnetic second harmonic generation," J. Magn. Magn. Mater. 198-199, 695-697 (1999).

23. K. J. Veenstra, A. V. Petukhov, E. Jurdik, and Th. Rasing "Strong surface state effects in nonlinear magneto-optical response of Ni(110)," Phys. Rev. Lett. 84, 2002-2005 $(2000)$.

24. A. Kirilyuk, Th. Rasing, M. A. M. Haast, and J. C. Lodder; "Probing structure and magnetism of $\mathrm{CoNi} / \mathrm{Pt}$ interfaces by nonlinear magneto-optics," Appl. Phys. Lett. 72, 2331$2333(1998)$.

25. M. Fiebig, D. Fröhlich, B. B. Krichevtsov, and R. V. Pisarev, "Second harmonic generation and magneticdipole-electric-dipole interference in antiferromagnetic $\mathrm{Cr}_{2} \mathrm{O}_{3}$," Phys. Rev. Lett. 73, 2127-2130 (1994).

26. M. Fiebig, D. Fröhlich, G. Sluyterman, and R. V. Pisarev, "Domain topography of antiferromagnetic $\mathrm{Cr}_{2} \mathrm{O}_{3}$ by 2ndharmonic generation," Appl. Phys. Lett. 22, 2906-2908 (1995).

27. V. V. Pavlov, R. V. Pisarev, A. Kirilyuk, and Th. Rasing, "Observation of a transversal nonlinear magneto-optical effect in thin magnetic garnet films," Phys. Rev. Lett. 78, 2004-2007 (1997).
28. A. Kirilyuk, V. V. Pavlov, R. V. Pisarev, and Th. Rasing, "Asymmetry of second harmonic generation in magnetic thin films under circular optical excitation," Phys. Rev. B 61, R3796-R3799 (2000).

29. V. N. Gridnev, V. V. Pavlov, R. V. Pisarev, A. Kirilyuk, and Th. Rasing, "Second harmonic generation in anisotropic magnetic films," Phys. Rev. B 63, 184407 (2001).

30. U. Pustogowa, W. Hübner, and K. H. Bennemann, "Theory for the nonlinear magneto-optical Kerr effect at ferromagnetic transition-metal surfaces," Phys. Rev, B 48, 8607 8618 (1993).

31. B. Koopmans, M. Groot Koerkamp, Th. Rasing, and Hugo van den Berg, "Observation of large Kerr angles in the nonlinear optical response from magnetic multilayers," Phys. Rev. Lett. 74, 3692-3695 (1995).

32. W. Hübner, "Theory of nonlinear surface magneto-optics for ferromagnetic nickel: Effects of band structure and matrix elements," Phys. Rev. B 42, 11553-11569 (1990).

33. T. Andersen and W. Hübner, "Substrate effects in the magneto-optical second-harmonic generation from first principles: Fe/Cu(001)," Phys. Rev. B 65, 174409 (2002).

34. L. Calmels, S. Crampin, J. E. Inglesfield, and Th. Rasing "Surface states and second harmonic generation at the (110) nickel surface from a first-principles calculation," Surf. Sci. 482, 1050-1055 (2001).

35. N. Bloembergen and P. S. Pershan, "Light waves at the boundary of nonlinear media," Phys. Rev. 128, 606-622 (1962).

36. J. E. Sipe, "New Green-function formalism for surface optics," J. Opt. Soc. Am. B 4, 481-489 (1987).

37. H. A. Wierenga, M. W. J. Prins, D. L. Abraham, and Th. Rasing, "Magnetization-induced optical second-harmonic generation: A probe for interface magnetism," Phys. Rev. B 50, 1282-1285 (1994).

38. H. A. Wierenga, M. W. J. Prins, and Th. Rasing, "Magnetization-induced optical second-harmonic generation from magnetic multilayers," Physica B 204, 281-286 (1995).

39. Handbook of Optical Constants of Solids, E. D. Palik, ed. (Academic, Orlando, Fla., 1985).

40. R. Atkinson and N. F. Kubrakov, "Boundary conditions in the simplest model of linear and second harmonic magneto-optical effects," Phys. Rev. B 65, 014432 (2002).

41. K. J. Veenstra, A. V. Petukhov, A. P. de Boer, and Th. Rasing, "Phase-sensitive detection technique for surface nonlinear optics," Phys. Rev. B 58, R16020-R16023 (1998).

42. St. Leute, Th. Lottermoser, and D. Fröhlich, "Nonlinear spatially resolved phase spectroscopy," Opt. Lett. 24, 1520-1522 (1999).

43. J. Hamrle, L. Polerecký, and J. Ferré, "Theory of secondharmonic generation from multilayer systems based on electric point-dipole radiation: Application to magnetic multilayers," Phys. Rev. B 68, 144401 (2003).

44. A. V. Petukhov, V. L. Brudny, W. L. Mochán, J. A. Maytorena, B. S. Mendoza, and Th. Rasing, "Energy conservation and the Manley-Rowe relations in surface nonlinearoptical spectroscopy," Phys. Rev. Lett. 81, 566-569 (1998).

45. K. Sato, A. Kodama, M. Miyamoto, A. V. Petukhov, K. Takanashi, S. Mitani, H. Fujimori, A. Kirilyuk, and Th. Rasing, "Anisotropic magnetization-induced second harmonic generation in $\mathrm{Fe}-\mathrm{Au}$ superlattices," Phys. Rev. B 64, 184427 (2001).

46. A. V. Petukhov, I. L. Lyubchanskii, and Th. Rasing, "Theory of nonlinear magneto-optical imaging of magnetic domains and domain walls," Phys. Rev. B، 56, 2680-2687 (1997).

47. R. Stolle, K. J. Veenstra, F. Manders, Th. Rasing, H. van den Berg, and N. Persat, "Breaking of time-reversal symmetry probed by optical second-harmonic generation," Phys. Rev. B 55, R4925-R4927 (1997).

48. K.-J. Veenstra, "Phase sensitive nonlinear magnetooptical spectroscopy" Ph.D. thesis (University of Nijmegen, The Netherlands, 2000).

49. Y. Uesu, S. Kurimura, and Y. Yamamoto, "Optical 2nd har- 
monic images of 9-degrees domain-structures in BaTiO, and periodically poled inverted antiparallel domains in $\mathrm{LiTaO}_{3}$, Appl. Phys. Lett. 66, 2165-2167 (1995).

50. S. Kurimura and Y. Uesu, "Application of the second harmonic generation microscope to nondestructive observation of periodically poled ferroelectric domains in quasiphase-matched wavelength converters," J. Appl. Phys. 81, 369-375 (1997).

51. S. I. Bozhevolnyi, J. M. Hvam, K. Pedersen, F. Laurell, H. Karlsson, T. Skettrup, and M. Belmonte, "Secondharmonic imaging of ferroelectric domain walls," Appl. Phys. Lett. 73, 1814-1816 (1998).

52. E. D. Mishina, N. E. Sherstyuk, E. Ph. Pevtsov, K. A. Vorotilov, A. S. Sigov, M. P. Moret, S. A. Rössinger, P. K. Larsen, and Th. Rasing, "Local probing of the polarization state in thin $\mathrm{Pb}(\mathrm{ZrTi}) \mathrm{O}_{3}$ films during polarization reversal," Appl. Phys. Lett. 78, 796-798 (2001).

53. M. Flörsheimer, M. Bösch, Ch. Brillert, M. Wierschem, and $H$. Fuchs, "Surface order and symmetry determined by second-harmonic microscopy," Supramol. Sci. 4, 255263 (1997).

54. M. Flörsheimer, M. Bösch, Ch. Brillert, M. Wierschem, and H. Fuchs, "Second-harmonic microscopy-A quantitative probe for molecular surface order;"Adv. Mater. 9, 1061 (1997).

55. K. Pedersen and S. I. Bozhevolnyi, "Second-harmonic generation scanning microscopy on domains in Al surfaces," Phys. Status Solidi A 175, 201-206 (1999).

56. J. Erland, S. I. Bozhevolnyi, K. Pedersen, J. R. Jensen, and J. M. Hvam, "Second-harmonic imaging of semiconduetor quantum dots," Appl. Phys. Lett. 77, 806-808 (2000).

57. I. I. Smolyaninov, A. V. Zayats, and C. C. Davis, "Nearfield second-harmonic imaging of ferromagnetic and ferroelectric materials," Opt. Lett. 22, 1592-1594 (1997).

58. S. I. Bozhevolnyi, B. Vohnsen, and K. Pedersen, "Nearfield optical microscopy of nonlinear susceptibilities," Opt. Commun. 150, 49-55 (1998).

59. A. V. Zayats and V. Sandoghdar, "Apertureless scanning near-field second-harmonic microscopy," Opt. Commun. $178,245-249$ (2000).

60. V. Kirilyuk, A. Kirilyuk, and Th. Rasing, "A combined nonlinear and linear magneto-optical microscopy," Appl. Phys. Lett. 70, 2306-2308 (1997)

61. A. Kirilyuk, Th. Rasing, R. Mégy, and P. Beauvillain "Nonlinear magneto-optical response from quantum well states in noble metals: double period and interface localization," Phys. Rev. Lett. 77, 4608-4611 (1996).

62. V. V. Pavlov, J. Ferré, P, Meyer, G. Tessier, P. Georges, A Brun, P. Beauvillain, and V. Mathet, "Linear and nonlinear magneto-optical studies of $\mathrm{Pt}-\mathrm{Co}-\mathrm{Pt}$ thin films," $J$. Phys.: Condens. Matter 13, 9867-9878 (2001).

63. A. Kirilyuk, V. Kirilyuk, and Th. Rasing, "Nonlinear magneto-optical imaging of interface magnetic structures," J. Magn. Magn. Mater. 198-199, 620-623 (1999).

64. M. D. Bijker; D. M. Donnet, and J. C. Lodder, "The effect of sputter pressure on the microstructure of $\mathrm{CoNi}-\mathrm{Pt}$ multilayers," J. Magn. Soc. Jpn. 20(S1), 407-409 (1996).

65. K. Kemnitz, K. Bhattacharya, J. M. Hicks, G. R. Pinto, K. B. Eisental, and T. F. Heinz, "The phase of 2nd-harmonic light generated at an interface and its relation to absolute molecular orientation," Chem. Phys. Lett. 131, 285-290 (1986).

66. R. Stolle, M. Loddoch, G. Marowsky, G. Berkovic, F. H. Kreuzer, and H. Leigeber, "Studies of nematic liquidcrystal cells using 2nd-harmonic phase measurements," Langmuir 11, 3251-3254 (1995).

67. R. K. Chang, J. Ducuing, and N. Bloembergen, "Relative phase measurement between fundamental and secondharmonic light," Phys. Rev, Lett. 15, 6-8 (1965).

68. S. Janz, D. J. Bottomley, H. M. van Driel, and R. S. Timsit, "Influence of steps on second-harmonic generation from vicinal metal surfaces," Phys. Rev. Lett. 66, 1201-1204 (1991).
69. A. K. Schmid and J. Kirsehner, "In situ observation of epitaxial growth of Co thin films on Cu(001)," Ultramicroscopy 42-44, 483-489 (1992).

70. It is easy to derive by use of the mirror symmetry operation with respect to the center plane of the film that the second-order susceptibilities of ideal mirrored interfaces are of equal amplitude but opposite phase.

71. O. A. Aktsipetrov, A. A. Nikulin, V. I. Panov, S. I. Vasil'ev, and A. V. Petukhov, "Electromagnetic mechanism of surface enhanced 2nd-harmonic generation by smooth silver electrodes and scanning tunneling microscopy," Solid State Commun. 76, 55-59 (1990).

72. K. Bal, H. A. M. van den Berg, A. Keen, and Th. Rasing, "Thermal stability of the antiferromagnetically coupled Co-Cu-Co stack," J. Appl. Phys. 89, 7508-7510 (2001).

73. K. Takanashi, S. Mitani, M. Sano, H. Fujimori, H. Nakajima, and A. Osawa, "Artificial fabrication of an L1/sub 0l-type ordered $\mathrm{FeAu}$ alloy by alternate monatomic deposition," Appl. Phys. Lett. 67, 1016-1018 (1995).

74. S. Mitani, K. Takanashi, H. Nakajima, K. Sato, R Schreiber, P. Grunberg, and H. Fujimori, "Structural and magnetic properties of Fe-noble metal monatomic multilayers equivalent to L1(0) ordered alloys," J. Magn. Magn. Mater. 156, 7-10 (1996).

75. K. Sato, J. Abe, H. Ikekame, K. Takanashi, S. Mitani, and H. Fujimori, "Magneto-optical characterization of $\mathrm{Fe}-\mathrm{Au}$ artificially ordered alloy,"J. Magn. Soc. Jpn. 20(S1), 35-40 (1996).

76. K. Sato, E. Takeda, M. Akita, M. Yamaguchi, K. Takanashi, S. Mitani, H. Fujimori, and Y. Suzuki, "Magnetooptical spectra of $\mathrm{Fe}-\mathrm{Au}$ artificial superlattices modulated by integer and noninteger atomic layers," J. Appl. Phys. 86, 4985-4996 (1999).

77. R. Vollmer, M. Straub, and J. Kirschner, "Second harmonic generation from the Cu(001) surface," Surf. Sci. 352, 684-688 (1996).

78. D. A. Koos, V. L. Shanon, and G. L. Richmond, "Surfacedipole and electric-quadrupole contributions to anisotropic second-harmonic generation from noble-metal surfaces," Phys. Rev. B 47, 4730-4734 (1993).

79. K. Pedersen and O. Keller, "Experimental study of phenomenological parameters describing optical 2ndharmonic generation in centrosymmetric materials," $\mathrm{J}$ Opt. Soc. Am. B 6, 2412-2419 (1989).

80. H. W. K. Tom, T. F. Heinz, and Y. R. Shen, "Secondharmonic reflection from silicon surfaces and its relation to structural symmetry," Phys. Rev. Lett. 51, 1983-1986 (1983)

81. M. Fiebig, D. Fröhlich, K. Kohn, St. Leute, Th. Lottermoser, V. V. Pavlov, and R. V. Pisarev, "Determination of the magnetic symmetry of hexagonal manganites by second harmonic generation," Phys. Rev. Lett. 84, 5620-5623 (2000).

82. A. Tsukamoto, K. Nakagawa, A. Itoh, A. van Etteger; A. Kirilyuk, and Th. Rasing, "Interface versus bulk magnetization reversal in TbFeCo thin films," to be published.

83. A. G. Banshchikov, A. V. Kimel, V. V. Pavlov, R. V. Pisarev, N. S. Sokolov, and Th. Rasing, "Generation of second optical harmonic and magneto-optical Kerr effect in ferromagnetic-semiconductor heterostructures $\mathrm{CaF}_{2}-\mathrm{MnAs}-\mathrm{Si}(111), "$ Phys. Solid State 42, 909-917 (2000).

84. H. Awano, S. Imai, M. Sekine, M. Tani, N. Ohta, K. Mitani, N. Takagi, H. Noguchi, and M. Kume, "20-nmdomain expansion readout by magnetic amplifying MO system (MAMMOS)," IEEE Trans. Magn. 36, 2261-2265 (2000).

85. Surface Polaritons, V. M. Agranovich and D. L. Mills, eds (North-Holland, Amsterdam, 1982).

86. V. I. Safarov, V. A. Kosobukin, C. Hermann, G. Lampel, and J. Peretti, "Magneto-optical effects enhanced by surface plasmons in metallie multilayer films," Phys. Rev. Lett. 73, 3584-3587 (1994).

87. C. K. Chen, A. R. B. de Castro, and Y. R. Shen, "Coherent second-harmonic generation by counterpropagating sur- 
face plasmons," Opt. Lett. 4, 393-394 (1979), and references cited therein.

88. V. V. Pavlov, G. Tessier, C. Malouin, P. Georges, A. Brun, D. Renard, P. Meyer, J. Ferré, and P. Beauvillain, "Observation of magneto-optical second-harmonic generation with surface plasmon excitation in ultrathin $\mathrm{Au}-\mathrm{Co}-\mathrm{Au}$ films," Appl. Phys. Lett. 75, 190-192 (1999).

89. D. Renard and G. Nihoul, "Crystal-structure of a magnetic cobalt layer deposited on a (111) gold surface determined by transmission electron-microscopy," Philos. Mag. B 55, $75-86$ (1987).

90. P. Guyot-Sionnest, W. Chen, and Y. R. Shen, "General considerations on optical second-harmonic generation from surfaces and interfaces," Phys. Rev. B 33, 8254-8263 (1986).

91. A. Liebsch and W. L. Schaich, "Second-harmonic generation at simple metal surfaces," Phys. Rev. B 40, 5401-5410 (1989).

92. V. A. Kosobukin, "Non-local magneto-optics of ultrathin ferromagnetic layers mediated by surface plasmonpolaritons," J. Magn. Magn. Mater. 153, 397-411 (1996).

93. 'T. V. Murzina, T. V. Misuryaev, A. F. Kravets, J. Güdde, D. Schuhmacher, G. Marowsky, A. A. Nikulin, and O. A. Akt sipetrov, "Nonlinear magneto-optical Kerr effect and plasmon-assisted SHG in magnetic nanomaterials exhibiting giant magnetoresistance," Surf. Sci. 482-485, 1101$1106(2001)$

94. X. D. Zhu, Hajo Suhr, and Y. R. Shen, "Surface vibrational spectroscopy by infrared-visible sum-frequency generation," Phys. Rev. B 35, 3047-3050 (1987).

95. P. Guyot-Sionnest, J. H. Hunt, and Y. R. Shen, "Sumfrequency vibrational spectroscopy of a Langmuir film Study of molecular orientation of a two-dimensional system," Phys. Rev. Lett. 59, 1597-1600 (1987).

96. A. L. Harris, C. E. D. Chidsey, N. J. Levinos, and D. N. Loiacono, "Monolayer vibrational spectroscopy on metal and semiconductor surfaces by infrared-visible sum generation," Chem. Phys. Lett. 141, 350-356 (1987).

97. E. V. Alieva, Y. E. Petrov, V. A. Yakovlev, V. A. Sychugov, E. R. Eliel, E. W. M. van der Ham, Q. H. F. Vrehen, and A. F. G. van der Meer, "Giant enhancement of sum-frequency generation upon excitation of a surface plasmonpolariton," JETP Lett. 66, 609-613 (1997).

98. A. Kirilyuk, G. M. H. Knippels, A. F. G. van der Meer, S. Renard, Th. Rasing, I. R. Heskamp, and J. C. Lodder, "Observation of strong magnetic effects in visible-infrared sum-frequency generation from magnetic structures," Phys. Rev. B 62, R783-R786 (2000).

99. D. Fröhlich, St. Leute, V. V. Pavlov, and R. V. Pisarev, "Nonlinear optical spectroscopy of the two-orderparameter compound $\mathrm{YMnO}_{3}$," Phys. Rev. Lett. 81, 32393242 (1998).

100. M. Fiebig, K. Miyano, Y. Tomioka, H. Kuwahara, Y.
Tokura, and K. Reimann, "Nonlinear magneto-optical properties of colossal magnetoresistive manganites," Phys. Rev. Lett. 86, 6002-6005 (2001).

101. M. Fiebig, D. Fröhlich, Th. Lottermoser, V. V. Pavlov, R. V. Pisarev, and H.-J. Weber; "Second harmonic generation in the centrosymmetric antiferromagnet NiO," Phys. Rev Lett. 87, 137202 (2001).

102. M. Fiebig, C. Degenhardt, and R. V. Pisarev, "Interaction of frustrated magnetic sublattices in $\mathrm{ErMnO}_{3}$," Phys. Rev. Lett. 88, 027203 (2002).

103. R. Vollmer; "Magnetization-induced second-harmonic generation from surfaces and ultrathin films," in Nonlinear Optics in Metals, K. H. Bennemann, ed. (Clarendon Press, Oxford, UK, 1998), p. 42.

104. T. A. Luce, W. Hübner, A. Kirilyuk, Th. Rasing, and K. H. Bennemann, "Theory for the nonlinear optical response of quantum-well states in ultrathin films," Phys. Rev. B 57, 7377-7384 (1998).

105. N. N. Dadoenkova, I. L. Lyubchanskii, M. I. Lyubchanskii, and Th. Rasing, "Nonlinear magneto-optical diffraction from periodic domain structures in magnetic films," Appl. Phys. Lett. 74, 1880-1882 (1999).

106. I. L. Lyubchanskii, N. N. Dadoenkova, M. I. Lyubchanskii, E. A. Shapovalov, and Th. Rasing, "Magnetic photonic crystals," J. Phys. D 36, R277-R287 (2003).

107. O. A. Aktsipetrov, "Nonlinear magneto-optics in magnetic nanoparticles," Colloids Surf. 202, 165-173 (2002).

108. Y. J. Ding and A. E. Kaplan, "Nonlinear magneto-optics of vacuum: Second-harmonic generation," Phys. Rev. Lett. 63, 2725-2728 (1989).

109. J. Hohlfeld, E. Matthias, R. Knorren, and K. H. Bennemann, "Nonequilibrium magnetization dynamics of nickel," Phys. Rev. Lett. 78, 4861-4864 (1997)

110. J. Güdde, U. Conrad, V. Jähnke, J. Hohlfeld, and E. Matthias, "Magnetization dynamics of $\mathrm{Ni}$ and $\mathrm{Co}$ films on Cu(001) and of bulk niekel surfaces," Phys. Rev. B 59, R6608-R6611 (1999).

111. H. Regensburger, R. Vollmer, and J. Kirschner, "Timeresolved magnetization-induced second-harmonic generation from the Ni(110) surface," Phys. Rev. B 61, 14716$14722(2000)$

112. T. M. Crawford, P. Kabos, and T. J. Silva, "Coherent control of precessional dynamies in thin film Permalloy," Appl. Phys. Lett. 76, 2113-2115 (2000).

113. T. J. Silva, M. R. Pufall, and P. Kabos, "Nonlinear magneto-optic measurement of flux propagation dynamics in thin Permalloy films," J. Appl. Phys. 91, 1066-1073 (2002).

114. Th. Gerrits, J. Hohlfeld, H. A. M. van den Berg, and Th. Rasing, "Ultrafast precessional magnetization reversal by picosecond magnetic field pulse shaping," Nature 418, 509-512 (2002). 